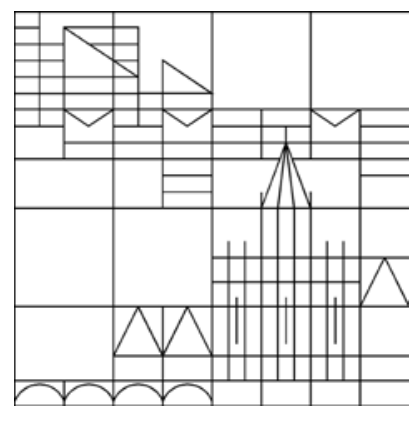

\title{
Bounds for the Range of a Complex Polynomial over a Rectangular Region
}

\author{
Jihad Titi \\ Jürgen Garloff
}

Konstanzer Schriften in Mathematik

Nr. 396, Dezember 2020

ISSN 1430-3558

Konstanzer Online-Publikations-System (KOPS)

URL: http://nbn-resolving.de/urn:nbn:de:bsz:352-2-2jh12ihlpabd2 



\title{
Bounds for the Range of a Complex Polynomial Over a Rectangular Region
}

\author{
Jihad Titi ${ }^{\mathrm{a}}$ and Jürgen Garloff ${ }^{\mathrm{b}, \mathrm{c}}$ \\ ${ }^{a}$ Department of Applied Mathematics and Physics, Palestine Polytechnic University \\ Hebron, Palestine \\ ${ }^{b}$ Department of Mathematics and Statistics, University of Konstanz, D-78464 Konstanz, \\ Germany \\ ${ }^{c}$ University of Applied Sciences / HTWG Konstanz, Institute for Applied Research, \\ Alfred-Wachtel-Str. 8, D-78462 Konstanz, Germany
}

\begin{abstract}
Matrix methods for the computation of bounds for the range of a complex polynomial and its modulus over a rectangular region in the complex plane are presented. The approach relies on the expansion of the given polynomial into Bernstein polynomials. The results are extended to multivariate complex polynomials and rational functions.
\end{abstract}

Keywords: Complex interval, Complex polynomial, Enclosure of the range, Bernstein polynomial, Multivariate complex polynomial, Multivariate rational function

2010 MSC: 65G40, 65E05

\section{Introduction}

Bounding the range of a function over a given region is an important task which is inherent in a remarkable variety of problems in mathematics and many of its applications. These include quantitative estimation of the remainder terms in numerical integration and differentiation, sensitivity analysis of systems, the certification of properties of function like monotonicity, convexity, and univalence, and branch and bound methods in global optimization, to name only a few. In this paper, we consider complex polynomials which arise in many areas such as control systems [5, Sections 3.1.7 and 3.1.8], image [12] and signal [16] processing, coding theory [18], and electrical networks [2]. The regions over which the range of such polynomials are 
sought are axis-aligned compact regions in the complex plane. The tool we are using is the expansion of the given polynomial into Bernstein polynomials. The convex hull of the coefficients of this expansion, the so-called Bernstein coefficients, provides an enclosure for the range of the given polynomial over the rectangular region. In contrast to the case of real polynomials, the use of the Bernstein polynomials for finding an enclosure for the range of a complex polynomial over a region in the complex plane has been considered in only a few papers so far. The first paper in this regard was [19] in which the range of a complex polynomial over the interval $[0,1]$ is enclosed. Rokne [20] and Grassmann and Rokne [10] extended this result to range enclosures over rectangles and discs in the complex plane. Furthermore, they considered enclosures for the range of complex polynomials with coefficients which are not exactly known but can located within rectangles or discs. Alternative methods to the Bernstein expansion include the so-called circular complex forms [21], [22], and [17, Chapter 2] which use circular arithmetic [6], [17, Section 1.3]. For the related problem of enclosing the range of a real polynomial over a simplex see [26] and references therein.

The organization of our paper is as follows. In the next section, we introduce the notation which is used throughout the paper. In Section 3, we first briefly recall the expansion of a multivariate real polynomial into Bernstein polynomials over a box and some of its fundamental properties. In the second part, we recall from [25], [27] a matrix method for the computation of the Bernstein coefficients. In Section 4, we present the expansion for a complex polynomial which is applied in Section 5 for finding an upper bound for the modulus of a polynomial. It turns out that the computation of the range of a complex polynomial over a rectangular region can be reduced to the calculation of the range over its boundary. In Section 6, some methods for the computation of the Bernstein coefficients of a complex polynomial are discussed which are extended in Section 8 to multivariate complex polynomials and in Section 9 to rational functions. In Section 7, we show how the Bernstein coefficients of a degree elevated expansion can be calculated from those of the real part of the lower degree expansion.

\section{Notation}

In this section, we introduce the notation that we are using throughout this paper. Let $n \in \mathbb{N}$ (set of the nonnegative integers) be the number of vari- 
ables. A multi-index $\left(i_{1}, \ldots, i_{n}\right) \in \mathbb{N}^{n}$ is abbreviated by $i$. In particular, we write 0 for $(0, \ldots, 0)$ and $e^{s}$ for the multi-ndex that has a 1 in position $s$ and 0's otherwise. Comparison between and arithmetic operations with multiindices are defined entry-wise. For the multi-index $i=\left(i_{1}, \ldots, i_{s}, \ldots, i_{n}\right)$ we define $i_{s, q}:=\left(i_{1}, \ldots, i_{s}+q, \ldots, i_{n}\right)$ and $i_{[s, q]}:=\left(i_{1}, \ldots, q, \ldots, i_{n}\right), s \in$ $\{1, \ldots, n\}, q \in \mathbb{Z}$. For $x=\left(x_{1}, \ldots, x_{n}\right) \in \mathbb{R}^{n}$, its monomials are defined as $x^{i}:=\prod_{s=1}^{n} x_{s}^{i_{s}}$. For $d=\left(d_{1}, \ldots, d_{n}\right) \in \mathbb{N}^{n}$ such that $i \leq d$, we use the compact notations $\sum_{i=0}^{d}:=\sum_{i_{1}=0}^{d_{1}} \ldots \sum_{i_{n}=0}^{d_{n}}$ and $\left(\begin{array}{c}d \\ i\end{array}\right):=\prod_{s=1}^{n}\left(\begin{array}{c}d_{s} \\ i_{s}\end{array}\right)$.

Let $\mathbb{I} \mathbb{R}$ be the set of compact, nonempty real intervals $\boldsymbol{x}=[\underline{x}, \bar{x}], \underline{x} \leq \bar{x}$. For $\boldsymbol{x} \in \mathbb{I R}$, we define the width of $\boldsymbol{x}$ by wid $\boldsymbol{x}:=\bar{x}-\underline{x}$. Let $\boldsymbol{x}, \boldsymbol{y} \in \mathbb{I} \mathbb{R}$. The Hausdorff distance between $\boldsymbol{x}$ and $\boldsymbol{y}$ is defined as

$$
\operatorname{dist}(\boldsymbol{x}, \boldsymbol{y}):=\max \{|\underline{x}-\underline{y}|,|\bar{x}-\bar{y}|\} \text {. }
$$

A box (also termed interval vector) is a vector and an interval matrix is a matrix with components from $\mathbb{I} \mathbb{R}$. Interval quantities (with the exception of intervals denoted by brackets) are written in bold. The width of a box is the maximum of the width of its components intervals.

The real and the imaginary parts of a complex number $z=x+y \mathrm{i} \in \mathbb{C}$, $\mathrm{i}^{2}=-1$, are denoted by $\operatorname{Re}(z)$ and $\operatorname{Im}(z)$. Let $z_{1}=x_{1}+y_{1} \mathrm{i}$ and $z_{2}=$ $x_{2}+y_{2} \mathrm{i} \in \mathbb{C}$. The line segment between $z_{1}$ and $z_{2}$ is the set

$$
\left\langle z_{1}, z_{2}\right\rangle:=\left\{z \in \mathbb{C} \mid z=(1-t) z_{1}+t z_{2}, 0 \leq t \leq 1\right\} .
$$

If $z_{1} \leq z_{2}$, i.e., $x_{1} \leq x_{2}$ and $y_{1} \leq y_{2}$, then we call

$$
\boldsymbol{z}=\left[z_{1}, z_{2}\right]:=\left\{x+y \mathrm{i} \mid x_{1} \leq x \leq x_{2}, y_{1} \leq y \leq y_{2}\right\}
$$

a (rectangular) complex interval. The set of all complex intervals is denoted by $\mathbb{I C}$. If $\boldsymbol{z} \in \mathbb{I} \mathbb{C}$, then it may also be written as $\boldsymbol{z}=\boldsymbol{x}+\boldsymbol{y i}$ with $\boldsymbol{x}, \boldsymbol{y} \in \mathbb{R}$. Geometrically, $\boldsymbol{z}$ is a compact axis-aligned region in the complex plane. Any complex number $z=x+y \mathrm{i}$ can be expressed as an interval $[x+y \mathrm{i}, x+y \mathrm{i}]$. Moreover, any real interval $[\underline{x}, \bar{x}]$ can be considered as a complex interval $\boldsymbol{z}=[\underline{x}+0 \mathrm{i}, \bar{x}+0 \mathrm{i}]$. The Cartesian product of complex intervals is called a complex box.

For the ease of presentation, we index all array entries starting from zero. 


\section{Real Bernstein Expansion}

\subsection{Bernstein Form Over the Unit Box}

In this section, we present fundamental properties of the Bernstein expansion over a box, e.g., [3, Subsection 5.1], [4], [7], [9], [19], that are employed throughout the paper. For simplicity we consider the unit box $\boldsymbol{u}:=[0,1]^{n}$, since any compact nonempty box $\boldsymbol{x}$ of $\mathbb{R}^{n}$ can be mapped affinely onto $\boldsymbol{u}$. Let $l \in \mathbb{N}^{n}, a_{j} \in \mathbb{R}, j=0, \ldots, l$, such that for $s=1, \ldots, n$

$$
l_{s}:=\max \left\{q \mid a_{j_{1}, \ldots, j_{s-1}, q, j_{s+1}, \ldots, j_{n}} \neq 0\right\} .
$$

Let $p$ be an $l$-th degree $n$-variate polynomial with the power representation

$$
p(x)=\sum_{j=0}^{l} a_{j} x^{j} .
$$

We expand $p$ into Bernstein polynomials of degree $d, d \geq l$, over $\boldsymbol{u}$ as

$$
p(x)=\sum_{j=0}^{d} b_{j}^{(d)} B_{j}^{(d)}(x),
$$

where $B_{j}^{(d)}$ is the $j$-th Bernstein polynomial of degree $d$, defined as

$$
B_{j}^{(d)}(x):=\left(\begin{array}{c}
d \\
j
\end{array}\right) x^{j}(1-x)^{d-j},
$$

and $b_{j}^{(d)}$ is the $j$-th Bernstein coefficient of $p$ of degree $d$ over $\boldsymbol{u}$ which is given by

$$
b_{j}^{(d)}=\sum_{i=0}^{j} \frac{\left(\begin{array}{l}
j \\
i
\end{array}\right)}{\left(\begin{array}{l}
d \\
i
\end{array}\right)} a_{i}, \quad 0 \leq j \leq d,
$$

with the convention that $a_{i}:=0$ if $i \geq l, i \neq l$, see, e.g., [19], [20]. We call (5) the Bernstein representation of $p$.

We arrange the Bernstein coefficients in a multidimensional array $B(\boldsymbol{u})=$ $\left(b_{j}^{(d)}\right)_{0 \leq j \leq d}$, the so-called Bernstein patch. The Bernstein coefficients provide lower and upper bounds for the range of $p$ over $\boldsymbol{u}$,

$$
\min _{j=0}^{d} b_{j}^{(d)} \leq p(x) \leq \max _{j=0}^{d} b_{j}^{(d)}, \text { for all } x \in \boldsymbol{u} .
$$


This property is called the range enclosure property. The interval spanned by the minimum and maximum Bernstein coefficients is called the Bernstein form of $p$ over $\boldsymbol{u}$ and denoted by $B^{(d)}(p, \boldsymbol{u})$. Equality holds in the left or the right inequalities of (8) if and only if the minimum or the maximum, respectively, is attained at a vertex of $B(\boldsymbol{u})$, i.e., if $j_{s} \in\left\{0, d_{s}\right\}, s=1, \ldots, n$. This condition is known as the vertex condition. Note that the Bernstein coefficients lying on the vertices of $B(\boldsymbol{u})$ are values of $p$ at the respective vertices of $\boldsymbol{u}$. More generally, the Bernstein coefficients on the $r$-dimensional faces of $\boldsymbol{u}, r=0,1, \ldots, n-1$, are just the Bernstein coefficients lying on the respective faces of $B(\boldsymbol{u})[9$, Lemma 2]. From the representation (7) the linearity of the Bernstein coefficients follows immediately: Let $p_{1}$ and $p_{2}$ be polynomials with the power representations (4) of maximum degree $l$. If $p=\alpha p_{1}+\beta p_{2}, \alpha, \beta \in \mathbb{R}$, then

$$
b_{j}^{(d)}(p)=\alpha b_{j}^{(d)}\left(p_{1}\right)+\beta b_{j}^{(d)}\left(p_{2}\right), \text { for all } 0 \leq j \leq d,
$$

where $b_{j}^{(d)}\left(p_{1}\right)$ and $b_{j}^{(d)}\left(p_{2}\right)$ are the $j$-th coefficients of the Bernstein expansions of degree $d$ of $p_{1}$ and $p_{2}$, respectively, $d \geq l$. The Bernstein form of $p$ over $\boldsymbol{u}$ is inclusion isotonic, i.e., if $\boldsymbol{u}$ is shrunk to a smaller box then the Bernstein enclosure shrinks, too, see [11, Theorem 3] for the univariate case and [8, Corollary 1] for the multivariate case.

We can improve the Bernstein form of $p$ over $\boldsymbol{u}(8)$ by elevating the degree $d$ of the Bernstein expansion or subdividing $\boldsymbol{u}$. Subdivision is more efficient than degree elevation since iteratively applied subdivision generates a sequence of enclosures which converges quadratically in the Hausdorff metric to the range of $p$ over $\boldsymbol{u}$, see, e.g., [24, Theorem 3.18], in contrast to linear convergence when degree elevation is applied, see Theorem 1.

Theorem 1. [19, Corollary], [7, Theorem 3] Let $p$ be a multivariate polynomial of degree $l$. Then, for $d \geq l$ with $d_{s} \geq 2, s=1, \ldots, n$, and $\kappa:=\max _{1 \leq s \leq n} d_{s}$ we have

$$
\operatorname{dist}\left(B^{(d)}(p, \boldsymbol{u}), p(\boldsymbol{u})\right) \leq \eta \frac{\kappa-1}{\kappa^{2}},
$$

where $\eta=\sum_{i=0}^{l} \sum_{s=1}^{n}\left(\max \left\{0, i_{s}-1\right\}\right)^{2}\left|a_{i}\right|$. 
Quadratic convergence of the Bernstein form to the range with respect to the width of the underlying box is provided in the next theorem.

Theorem 2. Let $\boldsymbol{y} \subseteq \boldsymbol{u}$ be a box. Under the hypothesis of Theorem 1, we have the following estimate

$$
\operatorname{dist}\left(B^{(d)}(p, \boldsymbol{y}), p(\boldsymbol{y})\right) \leq \omega \frac{\kappa-1}{\kappa^{2}}(\operatorname{wid}(\boldsymbol{y}))^{2},
$$

where

$$
\omega=\sum_{i=0}^{l} \sum_{s=1}^{n}\left(\max \left\{0, i_{s}-1\right\}\right)^{2} \sum_{t=i}^{l}\left(\begin{array}{l}
t \\
i
\end{array}\right)\left|a_{t}\right| .
$$

\subsection{Method for the Computation of the Bernstein Coefficients Over a Box}

We recall from [27] a method for the computation of the Bernstein coefficients of the $n$-variate polynomial $p$ given in (4) over the unit box $\boldsymbol{u}$. For the case of a general box see [27].

The superscript $c$ denotes the cyclic ordering of the sequence of the indices, i.e., the order of the indices of the entries of the array under consideration is changed cyclically. This means that the index in the first position is replaced by the index in the second one, the index in the second position by the one in the third, ..., the index in the $n$-th position by the one in the first position; see Figure 1 in [27] as an illustration in the trivariate case. So after $n$ cyclic orderings the sequence of the indices is again in its initial order. Note that in the bivariate case the cyclic ordering is just the usual matrix transposition.

The coefficients of $p$ are arranged in an $\left(l_{1}+1\right) \times l^{*}$ matrix $A$, where $l^{*}:=\prod_{s=2}^{n}\left(l_{s}+1\right)$. The correspondence between the coefficients $a_{j}$ of $p$ and the entry of $A$ in row $i$ and column $j$ is as follows:

$$
\begin{aligned}
& i=j_{1}, \\
& j=j_{2}+\sum_{s=3}^{n} j_{s}\left(l_{2}+1\right) \cdot \ldots \cdot\left(l_{s-1}+1\right) .
\end{aligned}
$$


Then $A$ can be represented as the matrix

$$
\begin{gathered}
{\left[\begin{array}{ccccccccccc}
a_{0,0,0, \ldots, 0} & a_{0,1,0, \ldots, 0} \ldots & a_{0, l_{2}, 0, \ldots, 0} & a_{0,0,1, \ldots, 0} & \ldots & a_{0, l_{2}, 1, \ldots, 0} & \ldots & a_{0,0, l_{3}, \ldots, 0} & \ldots & a_{0, l_{2}, l_{3}, \ldots, 0} \ldots \\
a_{1,0,0, \ldots, 0} & a_{1,1,0, \ldots, 0} \ldots & a_{1, l_{2}, 0, \ldots, 0} & a_{1,0,1, \ldots, 0} & \ldots & a_{1, l_{2}, 1, \ldots, 0} & \ldots & a_{1,0, l_{3}, \ldots, 0} & \ldots & a_{1, l_{2}, l_{3}, \ldots, 0} \ldots \\
\vdots & \vdots & \ldots & \vdots & \vdots & \ldots & \vdots & \ldots & \vdots & \ldots & \vdots \\
a_{l_{1}, 0,0, \ldots, 0} & a_{l_{1}, 1,0, \ldots, 0} \ldots & a_{l_{1}, l_{2}, 0, \ldots, 0} & a_{l_{1}, 0,1, \ldots, 0} & \ldots & a_{l_{1}, l_{2}, 1, \ldots, 0} & \ldots & a_{l_{1}, 0, l_{3}, \ldots, 0} & \ldots & a_{l_{1}, l_{2}, l_{3}, \ldots, 0} \ldots \\
\ldots & a_{0,0, l_{3}, \ldots, l_{n}} & a_{0,1, l_{3}, \ldots, l_{n}} & \ldots & a_{0, l_{2}, l_{3}, \ldots, l_{n}} \\
\ldots & a_{1,0, l_{3}, \ldots, l_{n}} & a_{1,1, l_{3}, \ldots, l_{n}} & \ldots & a_{1, l_{2}, l_{3}, \ldots, l_{n}} \\
\ldots & \vdots & \vdots & \ldots & & & & & & & \\
\ldots & a_{l_{1}, 0, l_{3}, \ldots, l_{n}} & a_{l_{1}, 1, l_{3}, \ldots, l_{n}} & \ldots & a_{l_{1}, l_{2}, l_{3}, \ldots, l_{n}}
\end{array}\right] .}
\end{gathered}
$$

The matrix $\Lambda(\boldsymbol{u})$ is obtained from $A$ by multiplying $a_{j}$ by $\left(\begin{array}{l}l \\ j\end{array}\right)^{-1}$. We put $\Lambda_{0}:=\Lambda(\boldsymbol{u})$ and define for $s=1, \ldots, n$

$$
\Lambda_{s}:=\left(P_{s} \Lambda_{s-1}\right)^{c}
$$

where $P_{s}$ is the lower triangular Pascal matrix,

$$
\left(P_{s}\right)_{i j}:= \begin{cases}\left(\begin{array}{l}
i \\
j
\end{array}\right), & \text { if } j \leq i, \\
0, & \text { otherwise. }\end{cases}
$$

In (14), the matrix multiplication is performed according to the factorization, e.g., [1, Lemma 1],

$$
P_{s}=\prod_{\mu=1}^{l_{s}} K_{\mu}^{s}
$$

where the bidiagonal matrices $K_{\mu}^{s}, \mu=1, \ldots, l_{s}$, are given by

$$
\left(K_{\mu}^{s}\right)_{i j}:= \begin{cases}1, & \text { if } i=j \\ 1, & \text { if } i=j+1, \quad l_{s}-\mu \leq j \leq l_{s}-1, \\ 0, & \text { otherwise. }\end{cases}
$$

Define for $s=1, \ldots, n, r:=s \bmod n$. Then for $s=1, \ldots, n$ the entry in position $\left(v_{1}, v_{2}\right)$ in $\Lambda_{s-1}$ becomes $\left(v_{1}^{\prime}, v_{2}^{\prime}\right)$ in $\Lambda_{s}$, where

$$
\begin{aligned}
& v_{1}^{\prime}=v_{2} \bmod \left(l_{r+1}+1\right), \\
& v_{2}^{\prime}=\left\lfloor\frac{v_{2}}{l_{r+1}+1}\right\rfloor+v_{1} \prod_{\substack{m=1, m \neq s, r}}^{n}\left(l_{m}+1\right) .
\end{aligned}
$$


The Bernstein patch $B(\boldsymbol{u})$ arranged accordingly in an $\left(l_{1}+1\right) \times l^{*}$ matrix, denoted by $\mathcal{B}(\boldsymbol{u})$, is given by $\Lambda_{n}$. Assuming that $\kappa=l_{s}$ for all $s=1, \ldots, n$, this method requires $n \kappa \frac{(\kappa+1)^{n}}{2}$ additions and $n(\kappa+1)^{n}$ multiplications for the computation of the Bernstein coefficients over the unit box $\boldsymbol{u}$, and needs $n \kappa(\kappa+1)^{n}+n$ additions and $3 n(\kappa+1)^{n}+2 n(\kappa-1)+n$ multiplications for a general box. A verified version of this method which is taking into account of all rounding errors as well as data uncertainties was recently implemented by Dr. Florian Bünger, Hamburg University of Technology, Germany. It is included in the version 12 of the MATLAB toolbox INTLAB [23].

\section{Univariate Complex Bernstein Expansion}

In this section, we recall from [20] the Bernstein representation of univariate complex polynomials over a complex interval in parallel to the one introduced in Subsection 3.1. Let $p$ be a univariate complex polynomial of degree $\kappa \in \mathbb{N}$ with the power representation

$$
p(z)=\sum_{j=0}^{\kappa} a_{j} z^{j}
$$

where $a_{j} \in \mathbb{C}, j=0, \ldots, \kappa$. For $z_{1} \in \mathbb{C}, p$ can be written as

$$
p(z)=\sum_{j=0}^{\kappa} c_{j}\left(z-z_{1}\right)^{j}
$$

where $c_{j}, j=0, \ldots, \kappa$, can be computed by

$$
c_{j}=\sum_{r=j}^{\kappa}\left(\begin{array}{l}
r \\
j
\end{array}\right)\left(z_{1}\right)^{r-j} a_{r} .
$$

For $z_{2} \in \mathbb{C}$, we expand $p$ into complex Bernstein polynomials of degree $d \geq \kappa$ over $\left\langle z_{1}, z_{2}\right\rangle$ as

$$
p(z)=\sum_{j=0}^{d} b_{j}^{(d)} B_{j}^{(d)}(z)
$$

where $B_{j}^{(d)}$ is the $j$-th Bernstein polynomial of degree $d$ over $\left\langle z_{1}, z_{2}\right\rangle$, defined as

$$
B_{j}^{(d)}(z):=\frac{\left(\begin{array}{c}
d \\
j
\end{array}\right)}{\left(z_{2}-z_{1}\right)^{d}}\left(z-z_{1}\right)^{j}\left(z_{2}-z\right)^{d-j}, \quad j=0, \ldots, d
$$


and $b_{j}^{(d)}$ is the $j$-th Bernstein coefficient of $p$ of degree $d$ over $\left\langle z_{1}, z_{2}\right\rangle$ given by

$$
b_{j}^{(d)}=\sum_{i=0}^{j} c_{i}\left(z_{2}-z_{1}\right)^{i} \frac{\left(\begin{array}{l}
j \\
i
\end{array}\right)}{\left(\begin{array}{c}
d \\
i
\end{array}\right)}, j=0, \ldots, d .
$$

We call (21) the Bernstein representation of $p$ over $\left\langle z_{1}, z_{2}\right\rangle$ [20]. Without loss of generality we assume in the sequel that $d=\kappa$.

Let $z_{1} \leq z_{2}$, where $z_{1}=x_{1}+y_{1} \mathrm{i}, z_{2}=x_{2}+y_{2}$ i. If $z=\left[z_{1}, z_{2}\right]$, it follows that $(x, y) \in \boldsymbol{x}$, where $\boldsymbol{x}:=\left[x_{1}, x_{2}\right] \times\left[y_{1}, y_{2}\right]$. Therefore, $p$ can be written as

$$
p(z)=p(x, y)=R(x, y)+I(x, y) \mathrm{i}
$$

where $R$ and $I$ are two real bivariate polynomials of degrees $l(R)$ and $l(I)$, respectively. Let $b_{j}^{\prime}, b_{j}^{\prime \prime}, j=0, \ldots, l$, denote the $j$-th Bernstein coefficients of $R$ and $I$ of degree $l=(\kappa, \kappa)$ over $\boldsymbol{x}$, respectively. We arrange these coefficients in Bernstein patches $B(R, \boldsymbol{x})=\left(b_{j}^{\prime}\right)_{0 \leq j \leq l}$ and $B(I, \boldsymbol{x})=\left(b_{j}^{\prime \prime}\right)_{0 \leq j \leq l}$, respectively. For later reference we introduce the index set $\mathcal{I}$ by

$$
\mathcal{I}:=\{(0,0),(0, \kappa),(\kappa, 0),(\kappa, \kappa)\}
$$

By expanding $R$ and $I$ into Bernstein polynomials of degree $l$ over $\boldsymbol{x}$, we obtain

$$
p(z)=p(x, y)=\sum_{j=0}^{l}\left(b_{j}^{\prime}+b_{j}^{\prime \prime} \mathrm{i}\right) B_{j}^{(l)}(x, y),
$$

where $B_{j}^{(l)}$ is the $j$-th Bernstein polynomial of degree $l$ over $\boldsymbol{x}$.

\section{An Upper Bound for the Modulus of a Polynomial}

Let $p$ be a complex polynomial of degree $\kappa$ as given in (18). We consider $p$ over a complex interval $\boldsymbol{z}$, such that $p$ is written in form (26) over $\boldsymbol{x}$. Since the Bernstein polynomials (22) are nonnegative on $\boldsymbol{z}$ and sum up to 1 [20], it follows from (26) that

$$
\begin{aligned}
|p(z)| & =\left|\sum_{j=0}^{l}\left(b_{j}^{\prime}+b_{j}^{\prime \prime} \mathrm{i}\right) B_{j}^{(l)}(x, y)\right| \leq \sum_{j=0}^{l}\left|b_{j}^{\prime}+b_{j}^{\prime \prime} \mathrm{i}\right| B_{j}^{(l)}(x, y) \\
& \leq \max _{j=0, \ldots, l}\left|b_{j}^{\prime}+b_{j}^{\prime \prime} \mathrm{i}\right|, \text { for all } z \in \boldsymbol{z} .
\end{aligned}
$$

As in the real case, see Subsection 3.1, the following vertex condition holds. 
Proposition 3. The upper bound in (27) is attained if and only if $\max _{j=0, \ldots, l} \mid b_{j}^{\prime}+$ $b_{j}^{\prime \prime} \mathrm{i}|=| b_{j^{\star}}^{\prime}+b_{j^{\star}}^{\prime \prime} \mathrm{i} \mid$ for some $j^{\star} \in \mathcal{I}$ given in $(25)$.

Proof. Assume that $\max _{j=0, \ldots, l}\left|b_{j}^{\prime}+b_{j}^{\prime \prime} \mathrm{i}\right|=\left|b_{j^{\star}}^{\prime}+b_{j^{\star}}^{\prime \prime} \mathrm{i}\right|$ for some $j^{\star} \in \mathcal{I}$. Then there exists $z^{\star}=x^{\star}+y^{\star} i \in \boldsymbol{z}$ with

$$
\left(x^{\star}, y^{\star}\right)=\left(x_{1}+\frac{j_{1}^{\star}}{\kappa}\left(x_{2}-x_{1}\right), y_{1}+\frac{j_{2}^{\star}}{\kappa}\left(y_{2}-y_{1}\right)\right)
$$

and we get

$$
p\left(z^{\star}\right)=p\left(x^{\star}, y^{\star}\right)=b_{j^{\star}}^{\prime}+b_{j^{\star}}^{\prime \prime} \mathrm{i} .
$$

It follows that

$$
\max _{z \in z}|p(z)|=\max _{j=0, \ldots, l}\left|b_{j}^{\prime}+b_{j}^{\prime \prime} \mathrm{i}\right|
$$

Conversely, suppose that for some $z^{\star} \in \boldsymbol{z}$ we have

$$
\left|p\left(z^{\star}\right)\right|=\max _{z \in z}|p(z)|=\max _{j=0, \ldots, l}\left|b_{j}^{\prime}+b_{j}^{\prime \prime} \mathrm{i}\right| .
$$

If all $\left|b_{j}^{\prime}+b_{j}^{\prime \prime} \mathrm{i}\right|, j=0, \ldots, l$, are equal, then the statement trivially holds. Otherwise, from (27) we get

$$
\left|p\left(z^{\star}\right)\right| \leq \sum_{j=0}^{l}\left|b_{j}^{\prime}+b_{j}^{\prime \prime} \mathrm{i}\right| B_{j}^{(l)}\left(x^{\star}, y^{\star}\right) \leq \max _{j=0, \ldots, l}\left|b_{j}^{\prime}+b_{j}^{\prime \prime} \mathrm{i}\right| .
$$

If $\left(x^{\star}, y^{\star}\right)$ is not a vertex of $\boldsymbol{x}$, then $B_{\hat{j}}^{(l)}\left(x^{\star}, y^{\star}\right) \in(0,1)$ for at least one $\hat{j} \in$ $\{0, \ldots, l\}$ such that the last inequality is strict, which provides a contradiction to $(28)$.

An upper bound for the modulus of $p$ over $\boldsymbol{z}$ can be obtained by the Maximum Modulus Principle Theorem. By employing this theorem it suffices to calculate the maximum modulus of the Bernstein coefficients of $p$ over the four line segments bordering $\boldsymbol{z}$. By the face value property, see Subsection 3.1 , these coefficients are identical to the ones lying on the respective edges of the Bernstein patch $B(p, \boldsymbol{x})$. Alternatively, the Bernstein coefficients of the four univariate polynomials obtained when $p$ is restricted to the edges of $\boldsymbol{z}$ can be separately computed. Another approach for finding an upper bound 
for the maximum modulus of $p$ is as follows: Let $H(x, y)$ be the bivariate polynomial of degree at most $(2 \kappa, 2 \kappa)$ defined by

$$
H(x, y):=|p(z)|^{2}=R^{2}(x, y)+I^{2}(x, y) .
$$

Again we consider the four univariate polynomials obtained when $H$ is restricted to the edges of $\boldsymbol{z}$ and calculate their Bernstein coefficients, where we have again two choices as above. Then we take the maximum square root instead of the maximum modulus. The question arises whether it requires less effort when the Bernstein coefficients of $R^{2}$ and $I^{2}$ are calculated directly from the Bernstein coefficients of $R$ and $I$ and then by the linearity of the Bernstein coefficients, see Subsection 3.1, their sum is formed or when $R$ and $I$ are firstly squared in their power representation, then the Bernstein coefficients of the squared polynomials are computed, and finally their sum is formed. From the results in [24, Section 3.7], [28, Section 4] it follows that the first method is more favorable. In all these variants, we recommend the method presented in Subsection 3.2 for the computation of the respective Bernstein coefficients.

\section{Enclosure of the Range of Univariate Complex Polynomials}

Let $\left\langle z_{1}, z_{2}\right\rangle$ be a line segment in the complex plane, and $p$ be a univarite polynomial of degree $\kappa$ as given in (18). Let $\mathcal{S}$ be the range of $p$ over $\left\langle z_{1}, z_{2}\right\rangle$, $\mathfrak{A}$ be the convex hull of $\mathcal{S}$, and $\mathfrak{A}_{d}$ be the convex hull of the Bernstein coefficients of $p$ of degree $d$ over $\left\langle z_{1}, z_{2}\right\rangle, d \geq \kappa$. By $\mathfrak{A}_{d}$ one obtains an enclosure of $\mathfrak{A}$.

Theorem 4. [19, Lemma 2 and Theorem 5], [20, Theorems 1 and 4] Let $\left\langle z_{1}, z_{2}\right\rangle$ be a line segment in the complex plane and $p$ be a complex univariate polynomial of degree $\kappa$, given in the Bernstein representation of degree $d$, $d \geq \kappa$. Then, the following relations are valid

$$
\begin{aligned}
& \mathfrak{A} \subseteq \mathfrak{A}_{d+1} \subseteq \mathfrak{A}_{d}, \\
& \mathfrak{A}=\cap_{d=\kappa}^{\infty} \mathfrak{A}_{d} .
\end{aligned}
$$

From (30), we conclude that the enclosure of the range of $p$ over a line segment provided by the Bernstein coefficients of $p$ can be improved by elevating the degree of the Bernstein expansion. Step by step degree elevation 
results in a sequence of enclosures which approximates the range of the polynomial better and better. However, the convergence of the sequence to the true range is only linear, see [19] and [20]. Quadratic convergence can be expected when subdivision is applied, see Subsection 3.1.

Now, we consider $p$ over a complex interval $\boldsymbol{z}=\left[z_{1}, z_{2}\right]$. Then the convex hull of the range of $p$ over $z$ is equal to the convex hull of the range of $p$ over the four line segments bordering $\boldsymbol{z}$. This is a consequence of the following theorem.

Theorem 5. [20, Lemma] If the complex function $f$ is analytic in a domain $R \subseteq \mathbb{C}$, continuous on $\operatorname{cl}(R)$, then $\operatorname{conv}(f(\operatorname{cl}(R)))$ is equal to $\operatorname{conv}(f(\partial R))$.

Hence, the problem of finding an enclosure for the range of $p$ of degree $\kappa$ given in (18) over the complex interval $\boldsymbol{z}$ is reduced to the problem of finding an enclosure for the convex hull of the range of $p$ over the four edges of $\boldsymbol{z}$. In the sequel we present some methods which can be used for the computation of the Bernstein coefficients over these edges. When comparing the required number of real arithmetic operations we count a complex addition as two real additions and a complex multiplication as two real additions and four real multiplications and assume that binomial coefficients are precomputed. Numerically, in general much sharper upper bounds for the range of $p$ over the edges can be obtained by solving global optimization problems for the four edge polynomials by employing the branch and bound algorithm using the Bernstein approach, see [14], [15], and [24]. Solving these problems yields upper bounds for the maximum value and bounds for the global maximizers of the objective functions (polynomials) of the related problems.

\subsection{Rokne's Method}

In this subsection, we briefly present Rokne's method [20]. Translating $\boldsymbol{z}$ from $z_{1}$ to the origin and from $z_{2}$ to the origin is required. Then the resulting polynomials, considered over the complex interval $\boldsymbol{z}^{\prime}:=\left[0, z_{2}-z_{1}\right]$, are referred to as $p_{1}$ and $p_{2}$, respectively. Let $c_{j}\left(p_{1}\right)$ and $c_{j}\left(p_{2}\right), j=0, \ldots, \kappa$, are the $j$-th coefficients of $p_{1}$ and $p_{2}$, respectively, such that

$$
p_{1}(z)=\sum_{j=0}^{\kappa} c_{j}\left(p_{1}\right) z^{j}
$$


and

$$
p_{2}(z)=\sum_{j=0}^{\kappa} c_{j}\left(p_{2}\right) z^{j}
$$

where for $j=0, \ldots, \kappa$, we have

$$
c_{j}\left(p_{1}\right)=\sum_{i=0}^{\kappa-j}\left(\begin{array}{c}
j+i \\
i
\end{array}\right) a_{j+i} z_{1}^{i},
$$

and

$$
c_{j}\left(p_{2}\right)=\sum_{i=0}^{\kappa-j}\left(\begin{array}{c}
j+i \\
i
\end{array}\right) a_{j+i}(-1)^{j} z_{2}^{i} .
$$

The two line segments

$$
\begin{aligned}
& I_{1}:=\left\langle 0, x_{2}-x_{1}\right\rangle, \\
& I_{2}:=\left\langle 0,\left(y_{2}-y_{1}\right) \mathrm{i}\right\rangle
\end{aligned}
$$

are edges of $\boldsymbol{z}^{\prime}$. Denote by $b_{j}\left(p_{m}, I_{h}\right)$ the $j$-th Bernstein coefficient of the polynomial $p_{m}$ over the $h$-th line segment $I_{h}, j=0, \ldots, \kappa, m, h \in\{1,2\}$, which can be computed by using (23). Then an upper bound for the range of $p$ over $\boldsymbol{z}$ is obtained by

$$
\max _{\substack{0 \leq j \leq \kappa \\ m, h=1,2}}\left\{\operatorname{Re}\left(b_{j}\left(p_{m}, I_{h}\right)\right)\right\}+\max _{\substack{0 \leq j \leq \kappa \\ m, h=1,2}}\left\{\operatorname{Im}\left(b_{j}\left(p_{m}, I_{h}\right)\right)\right\} \mathrm{i} .
$$

Replacing the max by min yields a lower bound. In Table 1, the number of the real arithmetic operations for the computation of the Bernstein coefficients of $p_{1}$ and $p_{2}$ by Rokne's method is presented (note that $z_{1}^{j}, z_{2}^{j}$, and $c_{j}, j=0, \ldots, \kappa$, are complex numbers). In total, Rokne's method requires $22 \kappa^{2}+36 \kappa-12$ real arithmetic operations (additions and multiplications/divisions). A matrix method based on Rokne's method is presented in Subsection 6.2.3.

\subsection{Proposed Matrix Methods}

In this subsection, we present three matrix methods for the computation of an enclosure for the range of $p$ over $\boldsymbol{z}$. 
Table 1: Number of real arithmetic operations for the computation of the Bernstein coefficients (BC) by Rokne's method

\begin{tabular}{|c|c|c|}
\hline Calculation of & number of additions & $\begin{array}{c}\text { number of } \\
\text { multiplications / divisions }\end{array}$ \\
\hline \hline $\begin{array}{c}z_{1}^{j}, z_{2}^{j}, c_{j}\left(p_{1}\right), \text { and } \\
c_{j}\left(p_{2}\right), j=0, \ldots, \kappa\end{array}$ & $2[2(\kappa-1)+2 \kappa(\kappa+1)]$ & $2[4(\kappa-1)+3 \kappa(\kappa+1)]$ \\
\hline $\begin{array}{c}\text { Bernstein coefficients of } p_{1} \\
\text { and } p_{2} \text { over } I_{1} \text { and } I_{2}\end{array}$ & $2[2(\kappa(\kappa+1))+1]$ & $2[2(2 \kappa(\kappa+1))+\kappa-1]$ \\
\hline total & $8 \kappa^{2}+12 \kappa-2$ & $14 \kappa^{2}+24 \kappa-10$ \\
\hline
\end{tabular}

\subsubsection{Method I}

Denote for $j=0, \ldots, \kappa$

$$
a_{j}^{\prime}:=\operatorname{Re}\left(a_{j}\right), \quad a_{j}^{\prime \prime}:=\operatorname{Im}\left(a_{j}\right) .
$$

Since $p$ can be represented as in (24), the two real bivariate polynomials $R$ and $I$ are given as

$$
R(x, y)=\sum_{\substack{j=0, j \text { even }}}^{\kappa} \sum_{i=0}^{\kappa-j}(-1)^{\frac{j}{2}}\left(\begin{array}{c}
i+j \\
j
\end{array}\right) a_{i+j}^{\prime} x^{i} y^{j}-\sum_{\substack{j=0, j \text { odd }}}^{\kappa} \sum_{i=0}^{\kappa-j}(-1)^{\frac{j+1}{2}}\left(\begin{array}{c}
i+j \\
j
\end{array}\right) a_{i+j}^{\prime \prime} x^{i} y^{j}
$$

and

$$
I(x, y)=\sum_{\substack{j=0, j \text { even }}}^{\kappa} \sum_{i=0}^{\kappa-j}(-1)^{\frac{j}{2}}\left(\begin{array}{c}
i+j \\
j
\end{array}\right) a_{i+j}^{\prime \prime} x^{i} y^{j}+\sum_{\substack{j=0, j \text { odd }}}^{\kappa} \sum_{i=0}^{\kappa-j}(-1)^{\frac{j-1}{2}}\left(\begin{array}{c}
i+j \\
j
\end{array}\right) a_{i+j}^{\prime} x^{i} y^{j} .
$$

Let $A_{R}$ and $A_{I}$ be the $(\kappa+1) \times(\kappa+1)$ real matrices that contain the coefficients of $R$ and $I$, respectively, such that for $j_{\nu}=0, \ldots, \kappa, \nu=1,2$, we have

$$
\left(A_{R}\right)_{j_{1} j_{2}}=\left\{\begin{array}{cc}
\left(\begin{array}{c}
j_{1}+j_{2} \\
j_{2}
\end{array}\right)(-1)^{\frac{j_{2}}{2}} a_{j_{1}+j_{2}}^{\prime}, & 0 \leq j_{1} \leq \kappa-j_{2}, j_{2} \text { even } \\
\left(\begin{array}{c}
j_{1}+j_{2} \\
j_{2}
\end{array}\right)(-1)^{\frac{j_{2}+1}{2}} a_{j_{1}+j_{2}}^{\prime \prime}, & 0 \leq j_{1} \leq \kappa-j_{2}, j_{2} \text { odd } \\
0, & \text { otherwise }
\end{array}\right.
$$

and

$$
\left(A_{I}\right)_{j_{1} j_{2}}=\left\{\begin{array}{cc}
\left(\begin{array}{c}
j_{1}+j_{2} \\
j_{2}
\end{array}\right)(-1)^{\frac{j_{2}}{2}} a_{j_{1}+j_{2}}^{\prime \prime}, & 0 \leq j_{2} \leq \kappa-j_{1}, j_{2} \text { even } \\
\left(\begin{array}{c}
j_{1}+j_{2} \\
j_{2}
\end{array}\right)(-1)^{\frac{j_{2}-1}{2}} a_{j_{1}+j_{2}}^{\prime}, & 0 \leq j_{2} \leq \kappa-j_{1}, j_{2} \text { odd } \\
0, & \text { otherwise }
\end{array}\right.
$$


We arrange the coefficients of $p$ in the $(\kappa+1) \times(\kappa+1)$ complex matrix $A_{p}$. Then from (24), (38), and (39), we get that

$$
\left(A_{p}\right)_{j_{1} j_{2}}=\left(A_{R}\right)_{j_{1} j_{2}}+\left(A_{I}\right)_{j_{1} j_{2}} \mathrm{i}, \quad j_{\nu}=0, \ldots, \kappa, \nu=1,2 .
$$

In Method I, the computation of the Bernstein coefficients of $R$ and $I$ over each edge of $\boldsymbol{x}$ is required. Let $E_{t}, t=1, \ldots, 4$, denote the $t$-th edge of $\boldsymbol{x}$, i.e.,

$$
E_{t}:=\left\{\begin{array}{cc}
{\left[x_{1}, x_{2}\right] \times\left\{y_{t}\right\},} & \text { for } t=1,2, \\
\left\{x_{t-2}\right\} \times\left[y_{1}, y_{2}\right], & \text { for } t=3,4 .
\end{array}\right.
$$

Denote $b_{j}\left(R, E_{t}\right)$ and $b_{j}\left(I, E_{t}\right)$ the $j$-th Bernstein coefficient of $R$ and $I$ of degree $(\kappa, \kappa)$ over the $t$-th edge of $\boldsymbol{x}$, respectively. These coefficients can be obtained by reducing $R$ and $I$ to two univariate polynomials over the edges given in (42) and using the matrix method presented in Subsection 3.2 as follows: Without loss of generality, we consider the case that we freeze $R$ over the edges $E_{3}$ and $E_{4}$. Let $R\left(x_{\rho}, y\right), \rho \in\{1,2\}$, be the polynomial which results when freezing $R$ on $E_{3}$, for $\rho=1$, or on $E_{4}$, for $\rho=2$. For $\rho=1,2$, assume that the coefficients of $R\left(x_{\rho}, y\right)$ are arranged in the row vector $A\left(R, x_{\rho}\right)$ of order $\kappa+1$. Then

$$
A\left(R, x_{\rho}\right)=H_{1}\left(x_{\rho}\right) \cdots H_{\kappa-1}\left(x_{\rho}\right) H_{\kappa}\left(x_{\rho}\right) A_{R},
$$

where the elementary bidiagonal matrix $H_{\mu}(x) \in \mathbb{R}^{\mu, \mu+1}, \mu=1, \ldots, \kappa, \rho=$ 1,2 , is defined by

$$
\left(H_{\mu}(x)\right)_{i j}:= \begin{cases}1, & i=j \\ x, & i=\mu, j=\mu+1 \\ 0, & \text { otherwise. }\end{cases}
$$

After that, the method from Subsection 3.2, is applied for the computation of the Bernstein coefficients of $R\left(x_{\rho}, y\right)$ over the respective edge. Note that for freezing $R$ on the edges $E_{1}$ and $E_{2}$, we firstly need to apply the cyclic ordering once, then employ the same method as in (43) to obtain $A\left(R, y_{\rho}\right)$, and then apply the cyclic ordering again. By (26), we have for $j=0, \ldots, l$, that the $j$-th Bernstein coefficient of $p$ over the $t$-th edge $E_{t}, t \in\{1, \ldots, 4\}$, is obtained as

$$
b_{j}\left(p, E_{t}\right)=b_{j}\left(R, E_{t}\right)+b_{j}\left(I, E_{t}\right) \mathrm{i}, j=0, \ldots, l .
$$


From the coefficients (45) we are able to get an enclosure of the range of $p$ over $\boldsymbol{z}$ as follows

$$
\left[\min _{\substack{0 \leq j \leq l \\ t=1, \ldots, 4}}\left\{b_{j}\left(R, E_{t}\right)\right\}+\min _{\substack{0 \leq j \leq l \\ t=1, \ldots, 4}}\left\{b_{j}\left(I, E_{t}\right)\right\} \mathrm{i}, \max _{\substack{0 \leq j \leq l \\ t=1, \ldots, 4}}\left\{b_{j}\left(R, E_{t}\right)\right\}+\max _{\substack{0 \leq j \leq l \\ t=1, \ldots, 4}}\left\{b_{j}\left(I, E_{t}\right)\right\} \mathrm{i}\right] .
$$

In Table 2, the number of real arithmetic operations for the computation of the Bernstein coefficients of $R$ and $I$ by Method I are presented.

Table 2: Number of real arithmetic operations for the computation of the Bernstein coefficients (BC) by Method I

\begin{tabular}{|c|c|c|}
\hline Calculation of & number of additions & $\begin{array}{c}\text { number of } \\
\text { multiplications / divisions }\end{array}$ \\
\hline \hline$A_{R}$ and $A_{I}$ by (40) and (41) & 0 & $2 \frac{\kappa(\kappa-1)}{2}$ \\
\hline $\begin{array}{c}\text { specification of } R \text { and } I \text { over } \\
\text { the four line segments }\end{array}$ & $4 \kappa(\kappa+1)$ & $4 \kappa(\kappa+1)$ \\
\hline $\begin{array}{c}\text { BC of } R \text { and } I \text { over the four } \\
\text { line segments by the method } \\
\text { from Subsection } 3.2\end{array}$ & $8[\kappa(\kappa+1)+1]$ & $8[3(\kappa+1)+2(\kappa-1)+1])$ \\
\hline total & $12 \kappa^{2}+12 \kappa+8$ & $5 \kappa^{2}+43 \kappa+16$ \\
\hline
\end{tabular}

The total number of real arithmetic operations for Method I is $17 \kappa^{2}+$ $55 \kappa+24$.

\subsubsection{Method II}

In contrast to Method I, here the Bernstein coefficients of $R$ and $I$ over the entire box $\boldsymbol{x}=\left[x_{1}, x_{2}\right] \times\left[y_{1}, y_{2}\right]$ are computed to obtain the ones over the four edges of $\boldsymbol{x}$. Here, we use the method presented in Subsection 3.2 starting from $A_{R}$ and $A_{I}$. Recall that the Bernstein coefficients of $R$ and $I$ over $\boldsymbol{x}$ are arranged in the Bernstein patches $B(R, \boldsymbol{x})$ and $B(I, \boldsymbol{x})$. Then the coefficients $b_{j}\left(R, E_{t}\right)$ and $b_{j}\left(I, E_{t}\right)$ over the edges $E_{t}, t=1, \ldots, 4$, given in (42), are obtained by taking those lying at the four faces of $B(R, \boldsymbol{x})$ and $B(I, \boldsymbol{x})$ (using the face values property of the Bernstein coefficients, see Subsection 3.1). Then an enclosure of the range of $p$ over $\boldsymbol{z}$ is obtained again by (45) and (46). In Table 3 the number of real arithmetic operations for the computation of the Bernstein coefficients of $R$ and $I$ by Method II is 
presented.

Table 3: Number of real arithmetic operations for the computation of the Bernstein coefficients (BC) by Method II

\begin{tabular}{|c|c|c|}
\hline Calculation of & number of additions & $\begin{array}{c}\text { number of } \\
\text { multiplications / divisions }\end{array}$ \\
\hline \hline$A_{R}$ and $A_{I}$ by (40) and (41) & 0 & $2 \frac{\kappa(\kappa-1)}{2}$ \\
\hline $\begin{array}{c}\text { BC of } R \text { and } I \text { by the method } \\
\text { from Subsection } 3.2\end{array}$ & $2\left[2 \kappa(\kappa+1)^{2}+2\right]$ & $2\left[6(\kappa+1)^{2}+4(\kappa-1)+2\right]$ \\
\hline total & $4 \kappa^{3}+8 \kappa^{2}+4 \kappa+4$ & $13 \kappa^{2}+31 \kappa+8$ \\
\hline
\end{tabular}

The total number of real arithmetic operations for Method II is $4 \kappa^{3}+$ $21 \kappa^{2}+35 \kappa+12$.

\subsubsection{Method III}

We present here a matrix method based on Rokne's method given in Subsection 6.1. Let $A_{p}^{\prime}, C_{p_{1}}$, and $C_{p_{2}} \in \mathbb{C}^{\kappa+1}$ contain the coefficients of $p, p_{1}$, and $p_{2}$, which are given in (33) and (34), respectively. $H_{1}$ and $H_{2}$ are two square upper triangular matrices of order $\kappa+1$ defined for $j_{\nu}=0, \ldots, \kappa, \nu=$ 1,2 , by

$$
\left(H_{m}\right)_{j_{1} j_{2}}:= \begin{cases}\left(\begin{array}{l}
j_{2} \\
j_{1}
\end{array}\right) z_{m}^{j_{2}-j_{1}}, & \text { if } j_{2} \geq j_{1} \\
0, & \text { otherwise }\end{cases}
$$

$D(z)$ is the diagonal matrix $\operatorname{diag}\left(1, z, \ldots, z^{\kappa}\right)$. Then, we have

$$
\begin{aligned}
& C_{p_{1}}=H_{1} A_{p}^{\prime}, \\
& C_{p_{2}}=D(-1) H_{2} A_{p}^{\prime} .
\end{aligned}
$$

It is easy to see that for $m=1,2$

$$
H_{m}=D\left(\frac{1}{z_{m}}\right) P^{T} D\left(z_{m}\right)
$$


where $P$ is the lower triangular Pascal matrix of order $\kappa+1$, see (15). Recall that $b_{j}\left(p_{m}, I_{h}\right)$ is the $j$-th Bernstein coefficient of the polynomial $p_{m}$ over the $h$-th line segment $I_{h}, j=0, \ldots, \kappa, m, h \in\{1,2\}$, see (35). Then, by using the method presented in Subsection 3.2 starting from $C_{p_{1}}$ and $C_{p_{2}}$, which are given in (48), respectively, one can compute the Bernstein patches that contain the coefficients of of $p_{1}$ and $p_{2}$ over $I_{1}$ and $I_{2}$. Finally, an enclosure of the range of $p$ over $\boldsymbol{z}$ is obtained from (36).

In Table 4 the number of real arithmetic operations for the computation of the Bernstein coefficients of $p_{1}$ over $I_{1}$ and $I_{2}$ by Method III is presented. The number of the operations for the computation of the Bernstein coefficients of $p_{2}$ over $I_{1}$ and $I_{2}$ is the same with the exception that the computation of $D\left(x_{2}-x_{1}\right)$ and $D\left(y_{2}-y_{1}\right)$ is not required, i.e., the number of multiplications/divisions is $2 \kappa-2$ less. The total number of arithmetic operations ${ }^{1}$ for Method III is $6 \kappa^{2}+72 \kappa-10$.

\subsection{Comparison of the Number of Arithmetic Operations and Examples}

In Table 5 the total number of arithmetic operations for the several methods are given which allow to draw the following conclusions: Rokne's method is superior to Method I for $\kappa \leq 5$, Method II is superior to Method I for $\kappa=1,2$, Method III is superior to Rokne's method for $\kappa \geq 3$, and Method III is superior to Methods I, II for all $\kappa$. Therefore, we recommend Rokne's method for $\kappa=1,2$ and Method III for $\kappa \geq 3$.

In Table 6, we present the timings of running the several methods for the seven polynomials listed in Appendix A. In the second column, the degree of the polynomials is displayed, which we have chosen as the degree of their Bernstein expansion, and in columns three to six and seven to ten the time (in $\mathrm{ms})$ for the computation of the Bernstein enclosure over the complex intervals $\boldsymbol{z}_{1}:=[0,1+\mathrm{i}]$ and $\boldsymbol{z}_{2}:=[-2-3 \mathrm{i}, 2-\mathrm{i}]$, respectively, is given. The enclosures themselves are listed in Table 9 in Appendix A. In [20], the range is considered over $z_{1}$ and in [10] over the disc of radius 1 centered at the origin. In both references, also an approximation of the contour of the respective range is presented which allows to judge upon the quality of the enclosure. As we

\footnotetext{
${ }^{1} A^{\star}=D(x) A$, such that $D(x):=\operatorname{diag}\left(1, x, x^{2}, \ldots, x^{\kappa}\right)$, where $x$ is either $x_{2}-x_{1}$ or $y_{2}-y_{1}$
} 
have mentioned in Subsection 3.1 and after Theorem 4, these enclosures can be tightened by degree elevation of the Bernstein expansion as well as by subdivision (preferred). The test polynomials $p_{i}, i=1,4,5$, are taken from [20], $p_{i}, i=2,3$, from [10], and $p_{i}, i=6,7$, from [21]. The timings presented in Table 6 clearly document the superiority of Method III. Finally, in Table 7 we quantify this superiority by giving the reduction of the timings for Method III compared to the ones for Rokne's method. The results in Table 6 are obtained on a laptop with Intel(R) Core(TM) i5-5200U CPU@ $3.30 \mathrm{GHz}$ 2.20 GHz, 16.00 GB RAM. The computations are done in Visual Studio 2010.

Table 4: Number of real arithmetic operations for the computation of the Bernstein coefficients (BC) of $p_{1}$ over $I_{1}$ and $I_{2}$ by Method III

\begin{tabular}{|c|c|c|}
\hline Calculation of & $\begin{array}{c}\text { number of } \\
\text { additions }\end{array}$ & $\begin{array}{c}\text { number of } \\
\text { multiplica- } \\
\text { tions } / \text { divisions }\end{array}$ \\
\hline \hline$D\left(z_{1}\right) A_{p}^{\prime}$ and $D\left(\frac{1}{z_{1}}\right)(\ldots)$ & $8 \kappa-4$ & $16 \kappa-8$ \\
\hline$P^{T}(\ldots)$ & $\kappa(\kappa+1)$ & 0 \\
\hline $\begin{array}{c}D\left(x_{2}-x_{1}\right) C_{p_{1}} \text { and } \\
D\left(y_{2}-y_{1}\right) C_{p_{2}}\end{array}$ & 0 & $2(\kappa-1)+2[2(\kappa+1)]$ \\
\hline BC of $p_{1}$ over $\left[0, x_{2}-x_{1}\right]$ and & $2\left[2\left(\frac{\kappa(\kappa+1)}{2}\right)\right]$ & $2[2(\kappa+1)]$ \\
\hline total & $3 \kappa^{2}+11 \kappa-4$ & $26 \kappa-2$ \\
\hline
\end{tabular}


Table 5: Total number of real arithmetic operations for the computation of an enclosure of the range of $p$ over $\boldsymbol{z}$ by the presented methods

\begin{tabular}{|c|c|}
\hline Calculation of & $\begin{array}{c}\text { total number of real } \\
\text { arithmetic operations }\end{array}$ \\
\hline \hline Rokne's method & $22 \kappa^{2}+36 \kappa-12$ \\
\hline Method I & $17 \kappa^{2}+55 \kappa+24$ \\
\hline Method II & $4 \kappa^{3}+21 \kappa^{2}+35 \kappa+12$ \\
\hline Method III & $6 \kappa^{2}+72 \kappa-10$ \\
\hline
\end{tabular}

Table 6: Time (in ms) required for computing the Bernstein enclosure over $\boldsymbol{z}_{1}$ and $\boldsymbol{z}_{2}$ by the presented methods

\begin{tabular}{|c|c||c|c|c|c|c|c|c|c|}
\hline $\begin{array}{r}\text { Test } \\
\text { case }\end{array}$ & $l$ & \multicolumn{4}{|c||}{$z_{1}=[0,1+\mathrm{i}]$} & \multicolumn{4}{|c|}{$z_{2}=[-2-3 \mathrm{i}, 2-\mathrm{i}]$} \\
\hline & & \multicolumn{1}{|c||}{$\begin{array}{c}\text { Rokne's } \\
\text { method }\end{array}$} & $\begin{array}{c}\text { Method } \\
\text { I }\end{array}$ & $\begin{array}{c}\text { Method } \\
\text { II }\end{array}$ & $\begin{array}{c}\text { Method } \\
\text { III }\end{array}$ & $\begin{array}{c}\text { Rokne's } \\
\text { method }\end{array}$ & $\begin{array}{c}\text { Method } \\
\text { I }\end{array}$ & $\begin{array}{c}\text { Method } \\
\text { II }\end{array}$ & $\begin{array}{c}\text { Method } \\
\text { III }\end{array}$ \\
\hline \hline$p_{1}$ & 3 & 0.417 & 0.683 & 0.730 & 0.378 & 0.423 & 0.786 & 0.808 & 0.410 \\
\hline$p_{2}$ & 4 & 0.546 & 0.887 & 0.896 & 0.456 & 0.595 & 1.014 & 1.081 & 0.505 \\
\hline$p_{3}$ & 4 & 0.544 & 0.887 & 0.899 & 0456 & 0.599 & 0.991 & 1.101 & 0.505 \\
\hline$p_{4}$ & 5 & 0.711 & 1.021 & 1.072 & 0.565 & 0.710 & 1.184 & 1.336 & 0.638 \\
\hline$p_{5}$ & 5 & 0.705 & 1.020 & 1.079 & 0.534 & 0.715 & 1.157 & 1.345 & 0.609 \\
\hline$p_{6}$ & 6 & 1.195 & 1.195 & 1.301 & 0.641 & 1.379 & 1.385 & 1.668 & 0.745 \\
\hline$p_{7}$ & 9 & 1.713 & 1.014 & 2.378 & 0.960 & 1.749 & 1.303 & 3.117 & 1.137 \\
\hline
\end{tabular}


Table 7: Reduction in time of Method III compared to Rokne's method

\begin{tabular}{|c|c|c|}
\hline Test case & $\begin{array}{c}\text { Reduction } \\
\text { over } \boldsymbol{z}_{1}\end{array}$ & $\begin{array}{c}\text { Reduction } \\
\text { over } \boldsymbol{z}_{2}\end{array}$ \\
\hline \hline$p_{1}$ & 9.35 & 3.07 \\
\hline$p_{2}$ & 16.48 & 15.13 \\
\hline$p_{3}$ & 16.18 & 15.69 \\
\hline$p_{4}$ & 20.53 & 10.14 \\
\hline$p_{5}$ & 24.26 & 14.83 \\
\hline$p_{6}$ & 46.36 & 45.98 \\
\hline$p_{7}$ & 43.96 & 34.99 \\
\hline
\end{tabular}

As an intermediate consequence of the inclusion isotonicity of the tensorial Bernstein form, see Subsection 3.1, we obtain the following theorem.

Theorem 6. The enclosures of the range of a complex polynomial given in subsections 6.1 and 6.2, see (36), (46), are inclusion isotonic.

\section{Computation of the Bernstein Coefficients of the Degree Ele- vated Expansion Using Those of the Real Part of Lower Degree}

Let $p$ be a univariate complex polynomial of degree $\kappa$ with its power representation given in (18). Recall that $p$ can be written as

$$
p(z)=p(x, y)=R(x, y)+I(x, y) \mathrm{i},
$$

where $R$ and $I$ are real bivariate polynomials of degrees $l(R)$ and $l(I)$, respectively. Without loss of generality, let us consider the unit box $\boldsymbol{u}$. We assume that $R$ is expanded into Bernstein polynomials of degree $l=(\kappa, \kappa)$ over $\boldsymbol{u}$, and that its Bernstein coefficients are arranged in the Bernstein patch $B(R, \boldsymbol{u})$. In this section, we present a matrix method in which $B(R, \boldsymbol{u})$ is used for the computation of the Bernstein patch $\tilde{B}(I, \boldsymbol{u})$ which comprises the Bernstein coefficients of degree $\tilde{l}:=(\kappa, \kappa+1)$ of $I$ over $\boldsymbol{u}$. Our method is based on the Cauchy-Riemann equations

$$
\frac{\partial R}{\partial x}=\frac{\partial I}{\partial y}, \quad \frac{\partial R}{\partial y}=-\frac{\partial I}{\partial x}
$$


and a method for the computation of the Bernstein coefficents of a multivariate polynomial from those of its partial derivatives [24, Section 3.6], [28, Section 5].

Let $b_{j}^{(\breve{l})}(R, \boldsymbol{u}), j=0, \ldots, \breve{l}$, be the $j$-th Bernstein coefficient of $R$ of degree $\breve{l}$ over $\boldsymbol{u}$, where $\breve{l}:=(\kappa+1, \kappa)$, obtained by elevating the degree of the Bernstein expansion of $R$ from $l$ to $\breve{l}$, i.e., elevating the degree with respect to $x$. Arrange these coefficients in the Bernstein patch $\breve{B}(R, \boldsymbol{u})$. Let $B\left(\frac{\partial R}{\partial x}, \boldsymbol{u}\right)$ be the Bernstein patch of $\frac{\partial R}{\partial x}$. From [9, formula (4)], we get that its entries can be computed by performing successive differences between the rows of $\breve{B}(R, \boldsymbol{u})$ and multiplying each entry by $\kappa+1$.

Since $B\left(\frac{\partial R}{\partial x}, \boldsymbol{u}\right)$ equals $B\left(\frac{\partial I}{\partial y}, \boldsymbol{u}\right)$, see (51), we may apply the mentioned method (starting from $B\left(\frac{\partial R}{\partial x}, \boldsymbol{u}\right)$ and using the partial derivative with respect to $y)$ to obtain the Bernstein patch $\tilde{B}(I, \boldsymbol{u})$ which contains the Bernstein coefficients of degree $\tilde{l}$ of $I$ over $\boldsymbol{u}$. Finally, we arrive at the Bernstein patch of $p$ of degree $\tilde{l}$ over $\boldsymbol{u}$ by elevation of the degree of the Bernstein expansion of $R$ from $l$ to $\tilde{l}$. In Figure 1 we present a flowchart of the procedure, where we give besides the Bernstein patches also their degrees. In a similar manner, we can also use the Bernstein expansion of degree $l$ of $I$ to obtain the Bernstein expansion of degree $\tilde{l}$ of $p$. In either case, the number of arithmetic operations is roughly the half of those which are required by Method II given in Subsection 6.2.2 to compute of the Bernstein coefficients of both the real and imaginary parts, see Table 3 . 


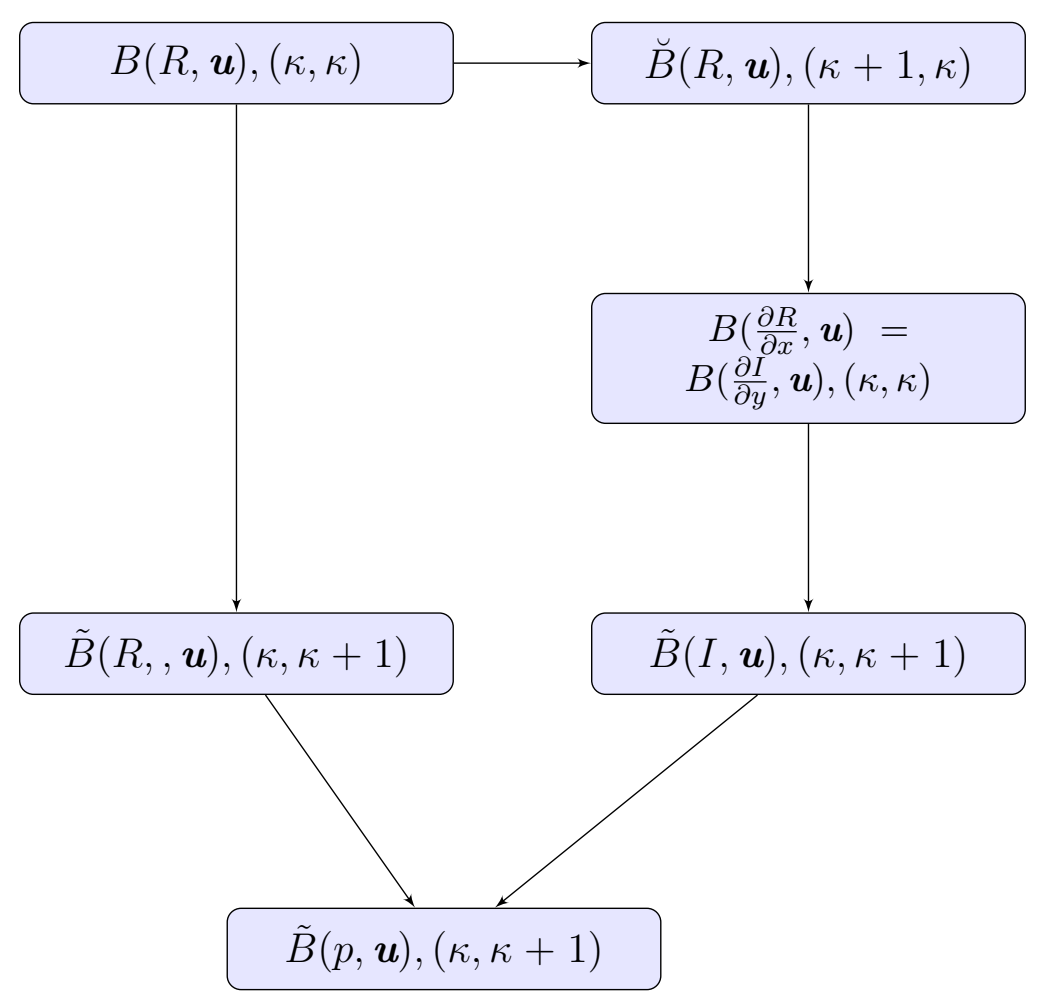

Figure 1: Flowchart of the procedure presented in Section 7

\section{Enclosure of the Range of Multivariate Complex Polynomials}

Let $z=\left(z_{1}, \ldots, z_{n}\right) \in \mathbb{C}^{n}$ be such that $z_{s}=x_{s}+y_{s} \mathrm{i}, \quad s=1, \ldots, n$. We set $x=\left(x_{1}, \ldots, x_{n}\right)$ and $y=\left(y_{1}, \ldots, y_{n}\right)$ and consider the complex box $\boldsymbol{z}:=\prod_{s=1}^{n}\left[z_{1, s}, z_{2, s}\right], z_{1, s}:=\alpha_{s}+\beta_{s} \mathrm{i}, z_{2, s}:=\gamma_{s}+\delta_{s} \mathrm{i}, s=1, \ldots, n$.

Let $p$ be a multivariate complex polynomial of degree $k^{\prime}=\left(k_{1}^{\prime}, \ldots, k_{n}^{\prime}\right)$, where $k^{\prime}$ is defined similarly as in (3), with the power representation

$$
p(z)=\sum_{j=0}^{k^{\prime}} a_{j} z^{j}
$$

where $a_{j} \in \mathbb{C}, j=0, \ldots, k^{\prime}$. Let $z \in \boldsymbol{z}$ be such that $x \in \prod_{s=1}^{n}\left[\alpha_{s}, \gamma_{s}\right]$ and $y \in \prod_{s=1}^{n}\left[\beta_{s}, \delta_{s}\right]$. For $s=1, \ldots, n$, we define $x_{s+n}:=y_{s}$, and rename $x:=$ 
$\left(x_{1}, \ldots, x_{2 n}\right) \in \mathbb{R}^{2 n}$. Substituting $z_{s}=x_{s}+x_{s+n} \mathrm{i}, s=1, \ldots, n$, into (52), allows us to represent $p$ over

$$
\boldsymbol{x}:=\prod_{s=1}^{n}\left[\alpha_{s}, \gamma_{s}\right] \times \prod_{s=1}^{n}\left[\beta_{s}, \delta_{s}\right]
$$

in the form

$$
p(z)=p(x)=R(x)+I(x) \text { i. }
$$

Expanding $R$ and $I$ into Bernstein polynomials of degree $d \geq k=$ $\left(k_{1}, \ldots, k_{2 n}\right)$ over $\boldsymbol{x}$ gives

$$
p(z)=p(x)=\sum_{j=0}^{d}\left(b_{j}^{\prime}+b_{j}^{\prime \prime} \mathrm{i}\right) B_{j}^{(d)}(x),
$$

where $b_{j}^{\prime}, b_{j}^{\prime \prime}, j=0, \ldots, d$, are the $j$-th Bernstein coefficient of $R$ and $I$ of degree $d$ over $\boldsymbol{x}$, respectively. Theorem 4 carries over to the multivariate case. In the remainder of this section we present two extensions of Methods I and II given in Subsections 6.2.1 and 6.2.2 for the computation of an enclosure of the range of $p$ over $z$. For simplicity, we choose in the sequel $d$ equal to the degree of $p$. We use the representation (55) of $p$. Let $A_{R}$ and $A_{I}$ be the two $\left(d_{1}+1\right) \times d^{\star \star}$ real matrices that contain the coefficients $a_{j}(R)$ and $a_{j}(I)$ of $R$ and $I$, respectively, where $d^{\star \star}:=\prod_{s=2}^{2 n}\left(d_{s}+1\right)$. Arrange the coefficients of $p$ in the $\left(d_{1}+1\right) \times d^{\star \star}$ complex matrix $A_{p}$, then from (54), we may represent the entries of $A_{p}$ for $j_{1}=0, \ldots, d_{1}$ and $j_{2}=0, \ldots, d^{\star \star}-1$ as

$$
\left(A_{p}\right)_{j_{1}, j_{2}}=\left(A_{R}\right)_{j_{1}, j_{2}}+\left(A_{I}\right)_{j_{1}, j_{2}} \text { i. }
$$

In both methods, the computation of $R$ and $I$ over the $4 n$ faces of $\boldsymbol{x}$ of dimension $2 n-1$ is required. In Methods I and II, this is accomplished in the way that is described in Subsection 6.2.1. Then an enclosure for the range of $p$ over $\boldsymbol{z}$ is obtained as in (46). In the sequel, we assume that $d_{s}=\kappa, s=1, \ldots, 2 n$, and that the coefficients of $R$ and $I$ are given and they are arranged in the matrices $A_{R}$ and $A_{I}$, respectively.

In Table 8, the number of real arithmetic operations for the computation of the Bernstein coefficients of $R$ and $I$ by Method I is presented. 
Table 8: Number of real arithmetic operations for the computation of the Bernstein coefficients (BC) by Method I

\begin{tabular}{|c|c|c|}
\hline Calculation of & number of additions & $\begin{array}{c}\text { number of } \\
\text { multiplications }\end{array}$ \\
\hline $\begin{array}{c}\text { specification of } R \text { and } I \text { over } \\
\text { the } 4 n \text { faces }\end{array}$ & $4 n \kappa(\kappa+1)^{2 n-1}$ & $4 n \kappa(\kappa+1)^{2 n-1}$ \\
\hline $\begin{array}{c}\text { BC of } R \text { and } I \text { over the } 4 n \\
\text { faces by the method from } \\
\text { Subsection } 3.2\end{array}$ & $\begin{array}{c}2\left[4 n\left[(2 n-1) \kappa(\kappa+1)^{2 n-1}+\right.\right. \\
2 n-1]]\end{array}$ & $\begin{array}{c}2\left[4 n\left[3(2 n-1)(\kappa+1)^{2 n-1}+\right.\right. \\
2(2 n-1)(\kappa-1)+2 n-1]]\end{array}$ \\
\hline total & $\begin{array}{c}4 n \kappa(\kappa+1)^{2 n-1}(4 n-1)+ \\
8 n(2 n-1)\end{array}$ & $\begin{array}{c}4 n(\kappa+1)^{2 n-1}(\kappa+12 n-6)+ \\
8 n(2 n-1)(2 \kappa-1)\end{array}$ \\
\hline
\end{tabular}

The total number of real arithmetic operations for Method I is $4 n(4 n \kappa+$ $12 n-6)(\kappa+1)^{2 n-1}+16 n \kappa(2 n-1)$. Method II requires $2\left[2 n \kappa(\kappa+1)^{2 n}+2 n\right]$ additions and $2\left[6 n(\kappa+1)^{2 n}+4 n(\kappa-1)+2 n\right]$ multiplications, in total $4 n(\kappa+$ $3)(\kappa+1)^{2 n}+8 n \kappa$. Thus, Method II is superior to Method I if $1 \leq \kappa \leq 4 n-2$ and Method I is superior to Method II if $\kappa \geq 4 n-1$.

It is worth to mention that Theorem 6 remains valid in the multivariate case. Assume that $p$ is a multivariate complex polynomial of degree $k^{\prime}$ as given in (52). For the rest of this section, we assume that $p$ is a multivariate complex polynomial of degree $k^{\prime}$ with its power representation given in (52), which allows the representation (55), where the corresponding real box $\boldsymbol{x}$ is defined in (53). As in Section 5, we obtain by $\max _{0 \leq j \leq d}\left|b_{j}^{\prime}+b_{j}^{\prime \prime} \mathrm{i}\right|$ an upper bound for $|p(z)|, z \in \boldsymbol{z}$, where $\boldsymbol{z}:=\prod_{s=1}^{n}[0,1+\mathrm{i}]$. The following theorem demonstrates the linear convergence of this bound to $\max _{z \in z}|p(z)|$ when the degree of the Bernstein expansion of $R$ and $I$ is elevated, see (55).

Theorem 7. Assume that $d_{s} \geq 2$ for $s=1, \ldots, 2 n$ and put $\kappa:=\max _{1 \leq s \leq 2 n} d_{s}$. Then

$$
\max _{0 \leq j \leq d}\left|b_{j}^{\prime}+b_{j}^{\prime \prime} \mathrm{i}\right|-\max _{z \in \boldsymbol{z}}|p(z)| \leq \Gamma \frac{\kappa-1}{\kappa^{2}}
$$


where

$$
\Gamma:=\sqrt{\eta_{R}^{2}+\eta_{I}^{2}}
$$

with

$$
\eta_{S}=\sum_{j=0}^{d} \sum_{s=1}^{2 n}\left(\max \left\{0, j_{s}-1\right\}\right)^{2}\left|a_{j}(S)\right|, S \in\{R, I\} .
$$

Proof. Assume that $\max _{0 \leq j \leq d}\left|b_{j}^{\prime}+b_{j^{\prime \prime}}^{\prime \prime}\right|=\left|b_{j^{\star}}^{\prime}+b_{j^{\star}}^{\prime \prime} \mathrm{i}\right|$ for some $0 \leq j^{\star} \leq d$. Then for the related grid point $x^{\star}=\frac{j^{\star}}{d} \in \boldsymbol{u}:=[0,1]^{2 n}$ we may estimate

$$
\begin{aligned}
\max _{0 \leq j \leq d}\left|b_{j}^{\prime}+b_{j}^{\prime \prime} \mathrm{i}\right|-\max _{z \in z}|p(z)| & \leq\left|b_{j^{\star}}^{\prime}+b_{j^{\star}}^{\prime \prime} \mathrm{i}\right|-\left|p\left(x^{\star}\right)\right| \\
& \leq\left|b_{j^{\star}}^{\prime}+b_{j^{\star}}^{\prime \prime} \mathrm{i}-p\left(x^{\star}\right)\right| \\
& =\left|\left(b_{j^{\star}}^{\prime}-R\left(x^{\star}\right)\right)+\left(b_{j^{\star}}^{\prime \prime}-I\left(x^{\star}\right)\right) \mathrm{i}\right| \\
& =\sqrt{\left|b_{j^{\star}}^{\prime}-R\left(x^{\star}\right)\right|^{2}+\left|b_{j^{\star}}^{\prime \prime}-I\left(x^{\star}\right)\right|^{2}} \\
& \leq \frac{\kappa-1}{\kappa^{2}} \sqrt{\eta_{R}^{2}+\eta_{I}^{2}} .
\end{aligned}
$$

The second inequality is obtained by using the reverse triangle inequality, the first equality is a consequence from (54), the last inequality and $\eta_{R}$ and $\eta_{I}$, that are given in (58), are provided by application of Theorem 1 to $R$ and $I$, respectively.

Remark 8. As in [19, Theorem 4] and [7, p.42], we obtain under the hypothesis of Theorem 7

$$
\left|p\left(\frac{j}{d}\right)-\left(b_{j}^{\prime}+b_{j}^{\prime \prime} \mathrm{i}\right)\right| \leq \Gamma \frac{\kappa-1}{\kappa^{2}}, \quad j=0, \ldots, d .
$$

In the next theorem, the quadratic convergence of the bounds to the maximum modulus when $\boldsymbol{z}$ is subdivided is stated. The proof is similar to the one of Theorem 7 and uses Theorem 2.

Theorem 9. Assume that $\boldsymbol{z}$ is subdivided into $\varsigma$ subboxes $\boldsymbol{z}_{m}, m=1, \ldots, \varsigma$, i.e., $\boldsymbol{z}=\cup_{m=1}^{\varsigma} \boldsymbol{z}_{m}$, where the interiors of the subboxes are disjoint. Let $\boldsymbol{x}_{m}$ denote the box that corresponds to $\boldsymbol{z}_{m}, m=1, \ldots, \varsigma$. Define by $b_{j}^{\prime}\left(\boldsymbol{x}_{m}\right)$ and 
by $b_{j}^{\prime \prime}\left(\boldsymbol{x}_{m}\right)$ the $j$-th Bernstein coefficients of $R$ and $I$ of degree $d$ over $\boldsymbol{x}_{m}$, respectively, where $j=0, \ldots, d$ and $m=1, \ldots, \varsigma$. Let $\mathscr{H}:=\max _{1 \leq m \leq \varsigma} \operatorname{wid}\left(\boldsymbol{x}_{m}\right)$. Then under the hypothesis of Theorem 7 we obtain the following estimate

$$
\max _{\substack{0 \leq j \leq d, m=1, \ldots, \varsigma}}\left|b_{j}^{\prime}\left(\boldsymbol{x}_{m}\right)+b_{j}^{\prime \prime}\left(\boldsymbol{x}_{m}\right) \mathrm{i}\right|-\max _{z \in \boldsymbol{z}}|p(z)| \leq \Omega \mathscr{H}^{2},
$$

where

$$
\Omega=\frac{\kappa-1}{\kappa^{2}} \sqrt{\omega_{R}^{2}+\omega_{I}^{2}}
$$

with

$$
\omega_{S}=\sum_{j=0}^{d} \sum_{s=1}^{2 n}\left(\max \left\{0, j_{s}-1\right\}\right)^{2} \sum_{t=j}^{k^{\prime}}\left(\begin{array}{l}
t \\
j
\end{array}\right)\left|a_{t}(S)\right|, S \in\{R, I\} .
$$

Denote by $B^{(d)}(R, \boldsymbol{x})$ and $B^{(d)}(I, \boldsymbol{x})$ the Bernstein forms of $R$ and $I$ of degree $d$ over $\boldsymbol{x}$. An enclosure for the range of $p$ over $\boldsymbol{z}$, which is denoted by $B^{(d)}(p, \boldsymbol{z})$, is provided by

$$
B^{(d)}(p, \boldsymbol{z})=B^{(d)}(R, \boldsymbol{x})+B^{(d)}(I, \boldsymbol{x}) \mathrm{i}
$$

i.e., we have

$$
p(\boldsymbol{z}) \subseteq B^{(d)}(p, \boldsymbol{z})
$$

Once the complex box $\boldsymbol{z}$ is subdivided into subboxes, we can get a tighter enclosure for the range of $p$ over $z$ as it is stated in the following theorem, which is a simple consequence of the inclusion isotonicity of the Bernstein form, see Subsection 3.1.

Theorem 10. Assume that $\boldsymbol{z}$ is subdivided into $\varsigma$ subboxes $\boldsymbol{z}_{m}, m=1, \ldots, \varsigma$, i.e., $\boldsymbol{z}=\cup_{m=1}^{\varsigma} \boldsymbol{z}_{m}$, where the interiors of the generated subboxes are disjoint. Let $B^{(d)}\left(p, \boldsymbol{z}_{m}\right)$ denote the enclosure for the range of $p$ of degree d over $\boldsymbol{z}_{m}, m=1, \ldots, \varsigma$, which is given by using (62) with $\boldsymbol{z}_{m}$ and $\boldsymbol{x}_{m}$. Then

$$
p(\boldsymbol{z}) \subseteq \cup_{m=1}^{\varsigma} B^{(d)}\left(p, \boldsymbol{z}_{m}\right) \subseteq B^{(d)}(p, \boldsymbol{z}) .
$$


By Theorems 4 and 5, the convex hull of the Bernstein coefficients of a univariate complex polynomial over an arbitrary complex box $\boldsymbol{z}$ provides an enclosure for the range of the polynomial over this box. Now, we consider the multivariate case. Let

$$
\begin{aligned}
\mathfrak{A} & :=\operatorname{conv}(p(\boldsymbol{z})) \\
\mathfrak{A}_{d} & :=\operatorname{conv}\left(\left\{b_{j}^{\prime}+b_{j}^{\prime \prime} \mathrm{i} \mid j=0, \ldots, d\right\}\right) .
\end{aligned}
$$

Let $z_{0} \in \boldsymbol{z}$ arbitrarily be chosen. Then for the related $x_{0} \in \boldsymbol{x}$ we may represent

$$
p\left(z_{0}\right)=\sum_{j=0}^{d}\left(b_{j}^{\prime}+b_{j}^{\prime \prime} \mathrm{i}\right) B_{j}^{(d)}\left(x_{0}\right) .
$$

Since $B_{j}^{(d)}\left(x_{0}\right), j=0, \ldots, d$, are nonnegative and form a partition of unity it follows from (66) that $p\left(z_{0}\right)$ is written as a convex combinations of $b_{j}^{\prime}+b_{j}^{\prime \prime} \mathrm{i}, j=$ $0, \ldots, d$. Hence, $p\left(z_{0}\right) \in \mathfrak{A}_{d}$ and we may conclude that $p(\boldsymbol{z}) \subseteq \mathfrak{A}_{d}$, and thus $\mathfrak{A} \subseteq \mathfrak{A}_{d}$

By elevating the degree of the Bernstein expansion of the given polynomial over a complex box $\boldsymbol{z}$, we obtain a sequence of enclosures that approximates $\mathfrak{A}$ better and better as the following theorem states. Its proof, see (72), reveals that this sequence converges linearly to $\mathfrak{A}$.

Theorem 11. Denote by $b_{j}^{\prime}(s)$ and $b_{j}^{\prime \prime}(s)$ the $j$-th Bernstein coefficients of $R$ and $I$, respectively, of degree $d+e^{s}$ over $\boldsymbol{x}, s=1, \ldots, 2 n$. Then for all $d \geq k$ and $s=1, \ldots, 2 n$, it holds that

$$
\begin{aligned}
& \mathfrak{A} \subseteq \mathfrak{A}_{d+e^{s}} \subseteq \mathfrak{A}_{d}, \\
& \mathfrak{A}=\cap_{d=k}^{\infty} \mathfrak{A}_{d} .
\end{aligned}
$$

Proof. We begin with the proof of (67a). Assume that $s \in\{1, \ldots, 2 n\}$ and suppose that $d_{s}=\kappa, s=1, \ldots, 2 n$. Then, we obtain by [3, p.391] that

$$
b_{j}^{\prime}(s)=\frac{j_{s} b_{j_{s,-1}}^{\prime}+\left(\kappa+1-j_{s}\right) b_{j}^{\prime}}{\kappa+1}
$$


and similarly for $b_{j}^{\prime \prime}(s)$ with the convention that $b_{j_{[s, w]}}^{\prime}=b_{j_{[s, w]}^{\prime \prime}}^{\prime \prime}=0$, where $w \in\left\{-1, d+e^{s}\right\}$. Then we get

$$
b_{j}^{\prime}(s)+b_{j}^{\prime \prime}(s) \mathrm{i}=\frac{j_{s}\left[b_{j_{s,-1}}^{\prime}+b_{j_{s,-1}}^{\prime \prime} \mathrm{i}\right]+\left(\kappa+1-j_{s}\right)\left[b_{j}^{\prime}+b_{j}^{\prime \prime} \mathrm{i}\right]}{\kappa+1},
$$

which means that $b_{j}^{\prime}(s)+b_{j}^{\prime \prime}(s) \mathrm{i}, j=0, \ldots, d+e^{s}$, are expressed as convex combinations of $b_{j}^{\prime}+b_{j}^{\prime \prime} \mathrm{i}, j=0, \ldots, d$. This implies that

$$
b_{j}^{\prime}(s)+b_{j}^{\prime \prime}(s) \mathrm{i} \in \mathfrak{A}_{d},
$$

and thus (67a). Now, we turn to the proof for (67b) following the one of Theorem 4 in [20]. Since $\mathfrak{A} \subseteq \mathfrak{A}_{d}$, for all $d \geq k$, we obtain the inclusion in (67b). Now assume that $z_{0} \in \boldsymbol{z}$ is such that $z_{0} \in \cap_{d=k}^{\infty} \mathfrak{A}_{d}$ and $z_{0} \notin \mathfrak{A}$. Then by the compactness of $\mathfrak{A}$, it holds that the smallest distance between $z_{0}$ and any $z \in \mathfrak{A}$, denoted by $\delta$, is positive. Choose $d \geq k$ such that $\frac{\kappa-1}{\kappa^{2}} \Gamma<\delta$, where $\Gamma$ is defined in (57). Since $z_{0} \in \mathfrak{A}_{d}$, there exist $\lambda_{j} \geq 0, j=0, \ldots, d$, with $\sum_{j=0}^{d} \lambda_{j}=1$, such that

$$
z_{0}=\sum_{j=0}^{d} \lambda_{j}\left(b_{j}^{\prime}+b_{j}^{\prime \prime} \mathrm{i}\right)
$$

Now using Remark 8, it follows that

$$
\begin{aligned}
\left|\sum_{j=0}^{d} \lambda_{j} p\left(\frac{j}{d}\right)-z_{0}\right| & \leq \sum_{j=0}^{d} \lambda_{j}\left|p\left(\frac{j}{d}\right)-\left(b_{j}^{\prime}+b_{j}^{\prime \prime} \mathrm{i}\right)\right| \\
& \leq \max _{0 \leq j \leq d}\left|p\left(\frac{j}{d}\right)-\left(b_{j}^{\prime}+b_{j}^{\prime \prime} \mathrm{i}\right)\right| \\
& \leq \Gamma \frac{\kappa-1}{\kappa^{2}},
\end{aligned}
$$

which is smaller than $\delta$, a contradiction to the assumption.

We are now taking into account the dependency of $\mathfrak{A}_{d}$ from the underlying box $\boldsymbol{z}$ by writing $\mathfrak{A}_{d}(\boldsymbol{z})=\operatorname{conv}\left(\left\{b_{j}^{\prime}(\boldsymbol{x})+b_{j}^{\prime \prime}(\boldsymbol{x}) \mathrm{i} \mid j=0, \ldots, d\right\}\right)$. The following theorem states that $\mathfrak{A}_{d}$ has the inclusion isotonicty property.

Theorem 12. For two complex boxes $\boldsymbol{z}_{1}, \boldsymbol{z}_{2}$, it holds that

$$
\text { if } \boldsymbol{z}_{1} \subseteq \boldsymbol{z}_{2} \text {, then } \mathfrak{A}_{d}\left(\boldsymbol{z}_{1}\right) \subseteq \mathfrak{A}_{d}\left(\boldsymbol{z}_{2}\right) .
$$


Proof. We obtain $\boldsymbol{z}_{1}$ from $\boldsymbol{z}_{2}$ by a finite sequence of subdivision steps. In each subdivision step we calculate the Bernstein coefficients of $R$ and $I$ over the corresponding real box by the de Casteljau algorithm, see, e.g., [3, Subsection 4.2]. Since in each step of this algorithm we are forming convex combinations, the Bernstein coefficients on a subdivided box are convex combinations of the Bernstein coefficients of the parent box and the proof is thus completed.

Let $E_{t}$ denote the $t$-th face of the faces of dimension $2 n-1$ of $\boldsymbol{x}$ given in (53) which are arranged in any order, $t=1, \ldots, 4 n$. Define by $b_{j}^{\prime}\left(E_{t}\right)$ and $b_{j}^{\prime \prime}\left(E_{t}\right)$ the $j$-th Bernstein coefficients of $R$ and $I$, respectively, of degree $d$, $d \geq k$, over $E_{t}$. Put

$$
\begin{aligned}
\mathfrak{A}^{\prime} & :=\operatorname{conv}(p(\partial \boldsymbol{z})), \\
\mathfrak{A}_{d}^{\prime} & :=\operatorname{conv}\left(\left\{b_{j}^{\prime}\left(E_{t}\right)+b_{j}^{\prime \prime}\left(E_{t}\right) \mathrm{i} \mid j=0, \ldots, d, t=1, \ldots, 4 n\right\}\right) .
\end{aligned}
$$

Then, the following theorem demonstrates that $\mathfrak{A}_{d}^{\prime}$ provides an enclosure for $\mathfrak{A}$, see (65a), which is not wider and often tighter than $\mathfrak{A}_{d}$ and $B^{(d)}(p, \boldsymbol{z})$ given in (62). For a given $z_{0} \in \partial \boldsymbol{z}$, let $E_{t}^{(0)}$ denote the $t$-th face of $\boldsymbol{x}$, where $z_{0}$ is lying. Define by $B_{j, t}^{(d)}$ the $j$-th Bernstein basis polynomial of degree $d$ over the $t$-th face of $\boldsymbol{x}, j=0, \ldots, d, t=1, \ldots, 4 n$.

Theorem 13. The following inclusions hold for all $d \geq k$

$$
\mathfrak{A} \subseteq \mathfrak{A}_{d}^{\prime} \subseteq \mathfrak{A}_{d}
$$

Proof. The second inclusion is obvious. Since by Theorem 5, the equality $\mathfrak{A}=\mathfrak{A}^{\prime}$ holds it is enough to show that $\mathfrak{A}^{\prime} \subseteq \mathfrak{A}_{d}^{\prime}$, where $\mathfrak{A}^{\prime}$ is defined in (74a). Let $z^{\prime} \in \mathfrak{A}^{\prime}$. Then, $z^{\prime}$ can be represented as

$$
z^{\prime}=\sum_{r=1}^{\rho} \lambda_{r} p\left(z_{r}\right)
$$

where $\sum_{r=1}^{\rho} \lambda_{r}=1, \lambda_{r} \geq 0$, and $z_{r} \in \partial z$, for all $r=1, \ldots, \rho$. Then

$$
z^{\prime}=\sum_{r=1}^{\rho} \sum_{j=0}^{d} \lambda_{r} B_{j, t}^{(d)}\left(z_{r}\right)\left(b_{j}^{\prime}\left(E_{t}^{(r)}\right)+b_{j}^{\prime \prime}\left(E_{t}^{(r)}\right) \mathrm{i}\right) .
$$

By the partition of unity property of $B_{j, t}^{(d)}\left(z_{r}\right), j=0, \ldots, d, r \in\{1, \ldots, \rho\}$, and $\sum_{r=1}^{\rho} \lambda_{r}=1$, it follows that $\sum_{r=1}^{\rho} \sum_{j=0}^{d} \lambda_{r} B_{j, t}^{(d)}\left(z_{r}\right)=1$. Thus, $z^{\prime}$ is written as a 
convex combination of $b_{j}^{\prime}\left(E_{t}^{(r)}\right)+b_{j}^{\prime \prime}\left(E_{t}^{(r)}\right) \mathrm{i}, j=0, \ldots, d, r=1, \ldots, \rho$, which proves $\mathfrak{A}^{\prime} \subseteq \mathfrak{A}_{d}^{\prime}$. Since

$\left\{b_{j}^{\prime}\left(E_{t}\right)+b_{j}^{\prime \prime}\left(E_{t}\right) \mathrm{i} \mid j=0, \ldots, d, t=1, \ldots, 4 n\right\} \subseteq\left\{b_{j}^{\prime}+b_{j}^{\prime \prime} \mathrm{i} \mid j=0, \ldots, d\right\}$,

see Subsection 3.1, it holds that $\mathfrak{A}_{d}^{\prime} \subseteq \mathfrak{A}_{d}$.

\section{Enclosure of the Range of Rational Complex Functions}

Let $q_{1}$ and $q_{2}$ be two $n$-variate complex polynomials. We may assume that the two polynomials have the same degree since otherwise we can elevate the degree of the Bernstein expansion of either polynomial by component where necessary to ensure that their Bernstein coefficients are of the same order. From (54), $q_{1}$ and $q_{2}$ can be written as

$$
q_{r}(z)=q_{r}(x)=R_{r}(x)+I_{r}(x) \mathrm{i}, \quad r \in\{1,2\},
$$

where $R_{r}, I_{r}, r=1,2$, are real $2 n$-variate polynomials. We consider the rational function

$$
f:=\frac{q_{1}}{q_{2}}
$$

over $\boldsymbol{z}$. From (78), $f$ can be written as

$$
f(z)=f(x)=Q_{1}(x)+Q_{2}(x) \mathrm{i}
$$

where

$$
Q_{r}(z)=Q_{r}(x)=\frac{Q_{r 1}(x)}{Q_{22}(x)}, \quad r=1,2
$$

with

$$
\begin{aligned}
& Q_{11}(x)=R_{1}(x) R_{2}(x)+I_{1}(x) I_{2}(x), \\
& Q_{21}(x)=I_{1}(x) R_{2}(x)-R_{1}(x) I_{2}(x), \\
& Q_{22}(x)=R_{2}^{2}(x)+I_{2}^{2}(x) .
\end{aligned}
$$

Then, for $j=0, \ldots, d$, let $b_{j}\left(Q_{11}\right), b_{j}\left(Q_{21}\right)$, and $b_{j}\left(Q_{22}\right)$ be the $j$-th Bernstein coefficient of $Q_{11}, Q_{21}$, and $Q_{22}$ of degree $d$ over $\boldsymbol{z}$, respectively, which can be 
computed directly from the Bernstein coefficents of the polynomials appearing on the right hand side of (82), see [24, Section 3.7]. We use the following notations

$$
b_{j}\left(Q_{r}\right):=\frac{b_{j}\left(Q_{r 1}\right)}{b_{j}\left(Q_{22}\right)}, j=0, \ldots, d ; r=1,2 .
$$

Under the assumption that the $b_{j}\left(Q_{22}\right)$ either all are positive or all negative, we obtain by [13, Theorem 3.1] that for all $x \in \boldsymbol{x}$

$$
\min _{0 \leq j \leq d} b_{j}\left(Q_{r}\right) \leq Q_{r}(x) \leq \max _{0 \leq j \leq d} b_{j}\left(Q_{r}\right), \quad r=1,2,
$$

and thus an enclosure for the range of $f$ over $\boldsymbol{z}$ is obtained by

$$
\left[\min _{0 \leq j \leq d} b_{j}\left(Q_{1}\right)+\min _{0 \leq j \leq d} b_{j}\left(Q_{2}\right) \mathrm{i}, \max _{0 \leq j \leq d} b_{j}\left(Q_{1}\right)+\max _{0 \leq j \leq d} b_{j}\left(Q_{2}\right) \mathrm{i}\right] .
$$

Example 14. We consider the univariate rational function $f(z)=\frac{1+2 z^{2}+z^{3}}{2-z+3 z^{2}}$ over $\boldsymbol{z}=[0.6-1.4 \mathrm{i}, 1.4-0.6 \mathrm{i}]$ [17, Example 2]. By the application of the method presented in this section, we obtain in $0.793 \mathrm{~ms}$ the enclosure [0.846$0.547 \mathrm{i}, 1.257-0.244 \mathrm{i}]$ for the range of $f$ over $\boldsymbol{z}$. The area of this enclosure is less than 0.131, whereas in [17] the application of the centered form to $f$ over the disc centered at $1-\mathrm{i}$ of radius 0.4 inscribed in $\boldsymbol{z}$ provides a disc as enclosure of the much larger area 3.141. An inner estimate for the range of $f$ over $\boldsymbol{z}$ (obtained by evaluation of $f$ at 5000 point in $\boldsymbol{z}$ ) is [1.068 $0.517 \mathrm{i}, 1.206-0.244 \mathrm{i}]$.

\section{Appendix A Description of the Polynomial Test Problems}

- $p_{1}(z)=(1+\mathrm{i})-(1+\mathrm{i}) z+(1+\mathrm{i}) z^{2}+\mathrm{i} z^{3} \quad$ [20, Example 4],

- $p_{2}(z)=(1+0.5 \mathrm{i}) z+0.5 \mathrm{i} z^{2}+0.6 z^{3}+(4+\mathrm{i}) z^{4} \quad$ [10, Example 2],

- $p_{3}(z)=1+\mathrm{i}+(0.4+\mathrm{i}) z^{2}+0.5 \mathrm{i} z^{3}+0.6 z^{4} \quad$ [10, Example 3],

- $p_{4}(z)=0.5+0.5 \mathrm{i}+(0.0032-0.0012 \mathrm{i}) z+(-0.006+0.012 \mathrm{i}) z^{2}+(0.02+$ $0.047 \mathrm{i}) z^{3}+(-0.3-0.15 \mathrm{i}) z^{4}+0.2 z^{5} \quad[20$, Example 5],

- $p_{5}(z)=0.5+0.5 \mathrm{i}-0.1476 z+(0.5-0.5 \mathrm{i}) z^{2}+\mathrm{i} z^{3}-(0.5+0.5 \mathrm{i}) z^{4}+0.2 z^{5}$ $\left[20\right.$, Example 6], ${ }^{2}$

\footnotetext{
${ }^{2}$ According to the deduction in [20, Example 6], the coefficients of $p_{5}$ are not correctly given therein.
} 
- $p_{6}(z)=0.8439-0.3312 \mathrm{i}+(0.1146-0.9919 \mathrm{i}) z+(0.3695-0.0198 \mathrm{i}) z^{2}+$ $(0.2170-0.0027 \mathrm{i}) z^{3}+(0.5042-0.9094 \mathrm{i}) z^{4}+(0.3355-0.0222 \mathrm{i}) z^{5}+$ $(0.1987-0.0227 \mathrm{i}) z^{6} \quad[21$, Example 3],

- $p_{7}(z)=0.2646-0.0352 \mathrm{i}+(0.6742-0.0056 \mathrm{i}) z+(0.1471-0.7941 \mathrm{i}) z^{2}+$ $(0.8776-0.2919 \mathrm{i}) z^{3}+(0.2863-0.9307 \mathrm{i}) z^{4}+(0.7096-0.0342 \mathrm{i}) z^{5}+$ $(0.1840-0.2115 \mathrm{i}) z^{6}+(0.8061-0.0060 \mathrm{i}) z^{7}+(0.0577-0.0711 \mathrm{i}) z^{8}+$ $(0.9947-0.6284 \mathrm{i}) z^{9} \quad[21$, Example 1]. 
Table 9: The Bernstein enclosure over $\boldsymbol{z}_{1}:=[0,1+\mathrm{i}]$ and $\boldsymbol{z}_{2}:=[-2-3 \mathrm{i}, 2-\mathrm{i}]$, respectively.

\begin{tabular}{|c|c|c|}
\hline $\begin{array}{l}\text { Test } \\
\text { case }\end{array}$ & $\begin{array}{c}\text { Bernstein enclosure } \\
\text { over } \boldsymbol{z}_{1}\end{array}$ & $\begin{array}{l}\text { Bernstein enclosure over } \\
\boldsymbol{z}_{2}\end{array}$ \\
\hline$p_{1}$ & {$[-3-2 \mathrm{i}, 2+2.333 \mathrm{i}]$} & {$[-54.667-61 \mathrm{i}, 24+59 \mathrm{i}]$} \\
\hline$p_{2}$ & $\begin{array}{c}{[-17.7-8.575 \mathrm{i}, 5.6+} \\
10.317 \mathrm{i}]\end{array}$ & $\begin{array}{c}{[-614.1-610.9 \mathrm{i}, 677.5+} \\
698.2 \mathrm{i}]\end{array}$ \\
\hline$p_{3}$ & $\begin{array}{c}{[-4.4-1.383 \mathrm{i}, 2+} \\
3.683 \mathrm{i}]\end{array}$ & $\begin{array}{c}{[-79.9-83.7 \mathrm{i}, 78.767+} \\
67.7 \mathrm{i}]\end{array}$ \\
\hline$p_{4}$ & $\begin{array}{c}{[0.254+} \\
0.236 \mathrm{i}, 0.890+0.837 \mathrm{i}]\end{array}$ & $\begin{array}{c}{[-158.175-} \\
138.742 \mathrm{i}, 132.485+ \\
175.593 \mathrm{i}]\end{array}$ \\
\hline$p_{5}$ & $\begin{array}{c}{[0.450+} \\
0.450 \mathrm{i}, 0.602+0.602 \mathrm{i}]\end{array}$ & $\begin{array}{c}{[-227.722-} \\
204.056 \mathrm{i}, 152.722+ \\
294.344 \mathrm{i}]\end{array}$ \\
\hline$p_{6}$ & $\begin{array}{c}{[-2.068-} \\
2.300 \mathrm{i}, 3.533+2.376 \mathrm{i}]\end{array}$ & $\begin{array}{c}{[-389.225-} \\
529.526 \mathrm{i}, 570.424+ \\
475.814 \mathrm{i}]\end{array}$ \\
\hline$p_{7}$ & $\begin{array}{c}{[-7.481-} \\
13.160 \mathrm{i}, 28.842+ \\
15.356 \mathrm{i}]\end{array}$ & $\begin{array}{c}{[-117250.429-} \\
117822.949 \mathrm{i}, 120539.093+ \\
110938.291 \mathrm{i}]\end{array}$ \\
\hline
\end{tabular}

\section{References}

[1] Alonso P., Delgado J., Gallego R., Peña J.M.: Conditioning and accurate computations with Pascal matrices. J. Comput. Appl. Math. 252, 21-26 (2013)

[2] Dòria-Cerezo A., Bodson M.: Design of controllers for electrical power 
systems using a complex root locus method. IEEE Trans. Industrial Electronics 63(6), 3706-3716 (2016)

[3] Farouki R.T.: The Bernstein polynomial basis: A centennial retrospective. Comput. Aided Geom. Design 29, 379-419 (2012)

[4] Fischer H.C.: Range computation and applications. In: Ullrich C. (ed.) Contributions to Computer Arithmetic and Self-Validating Numerical Methods, J.C. Baltzer, Amsterdam, pp. 197-211 (1990)

[5] Franklin G.F., Powell J.D., Emami-Naeini A.: Feedback Control of Dynamic Systems, eighth edition, Pearson, Upper Saddle River, N.J. (2019)

[6] Gargantini I., Henrici P.: Circular arithmetic and the determination of polynomial zeros. Numer. Math. 18, 305-320 (1972)

[7] Garloff J.: Convergent bounds for the range of multivariate polynomials. In: Nickel K. (ed.), Interval Mathematics 1985. Lecture Notes in Computer Science, vol. 212, Springer, Berlin, Heidelberg, pp. 37-56 (1986)

[8] Garloff J., Jansson C., Smith A.P.: Inclusion isotonicity of convexconcave extensions for polynomials based on Bernstein expansion. Computing 70, 111-119 (2003)

[9] Garloff J., Smith A.P.: Solution of systems of polynomial equations by using Bernstein expansion. In: Alefeld, G., Rump, S., Rohn, J., and Yamamoto J. (eds.) Symbolic Algebraic Methods and Verification Methods, Springer, pp. 87-97 (2001)

[10] Grassmann E., Rokne J.: The range of values of a circular complex polynomial over a circular complex interval. Computing 23, 139-169 (1979)

[11] Hong H., Stahl, V.: Bernstein form is inclusion monotone. Computing 55, 43-53 (1995)

[12] Li S., Lee M.-C., Pun C.-M.: Complex Zernike moments features for shape-based image retrieval. IEEE Trans. Systems, Man, Cybernetics Part A: Systems and Humans 39(1), 227-237 (2009)

[13] Narkawicz A., Garloff J., Smith A.P., Muñoz C.A.: Bounding the range of a rational function over a box. Reliab. Comput. 17, 34-39 (2012) 
[14] Nataraj P.S.V., Arounassalame M.: A new subdivision algorithm for the Bernstein polynomial approach to global optimization. Int. J. Automat. and Comput. 4, 342-352 (2007)

[15] Nataraj P.S.V., Arounassalame M.: Constrained global optimization of multivariate polynomials using Bernstein branch and prune algorithm. J. Global Optim. 49, 185-212 (2011)

[16] Oppenheim A.V., Willsky A.S., with Hamid S.: Signal and Systems, 2nd edition, Pearson Education Ltd, Harlow, UK (2014)

[17] Petković M.S., Petković L.D.: Complex Interval Arithmetic and Its Applications, Series Math. Res. vol. 105, Wiley-VCH, Berlin, Weinheim et al. (1998)

[18] Pistone G., Rogantin M.P.: Indicator function and complex coding for mixed fractional factorial designs. J. Statist. Plann. Inference 138(3), 787$802(2008)$

[19] Rivlin T.J.: Bounds on a polynomial. J. Res. Nat. Bur. Standards 74 (B), 47-54 (1970)

[20] Rokne J.: The range of values of a complex polynomial over a complex interval. Computing 22, 153-169 (1979)

[21] Rokne J., Wu T.: The circular complex centered form. Computing 28, 17-30 (1982)

[22] Rokne J., Wu T.: A note on the circular complex centered form. Computing 30, 201-211 (1983)

[23] Rump S.M.: INTLAB-INTerval LABoratory. In: Csendes, T. (ed.) Developments in Reliable Computing, Kluwer Acad. Publ., Dordrecht, pp. 77-104 (1999)

[24] Titi J.: Matrix methods for the tensorial and simplicial Bernstein forms with application to global optimization, dissertation, University of Konstanz, Konstanz, Germany (2019), available at http://nbnresolving.de/urn:nbn:de:bsz:352-2-k106crqmste71 
[25] Titi J., Garloff J.: Fast determination of the tensorial and simplicial Bernstein forms of multivariate polynomials and rational functions. Reliab. Comput. 25, 24-37 (2017)

[26] Titi J., Garloff J.: Matrix methods for the simplicial Bernstein representation and for the evaluation of multivariate polynomials. Appl. Math. Comput. 315, 246-258 (2017)

[27] Titi J., Garloff J.: Matrix methods for the tensorial Bernstein form. Appl. Math. Comput. 346, 254-271 (2019)

[28] Titi J., Garloff J.: Symbolic-numeric computation of the Bernstein coefficients of a polynomial from those of one of its partial derivatives and of the product of two polynomials. In: Boulier F., England M., Sadykov T.M., Vorozhtsov E.V. (eds.) Computer Algebra in Scientific Computing. CASC 2020. Lecture Notes in Computer Science, vol. 12291, Springer, Cham, pp. 583-599 (2020) 